\title{
Monitoring and Control of a Continuous Grignard Reaction for the Synthesis of an Active Pharmaceutical Ingredient Intermediate Using Inline NIR spectroscopy
}

Cervera Padrell, Albert Emili; Nielsen, Jesper; Jønch Pedersen, Michael; Muller Christensen, Kim; Mortensen, Asmus R.; Skovby, Tommy; Dam-Johansen, Kim; Kiil, Søren; Gernaey, Krist V.

\section{Published in:}

Organic Process Research and Development

Link to article, DOI:

10.1021/op2002563

Publication date:

2012

Document Version

Publisher's PDF, also known as Version of record

Link back to DTU Orbit

Citation (APA):

Cervera Padrell, A. E., Nielsen, J., Jønch Pedersen, M., Muller Christensen, K., Mortensen, A. R., Skovby, T., Dam-Johansen, K., Kiil, S., \& Gernaey, K. V. (2012). Monitoring and Control of a Continuous Grignard Reaction for the Synthesis of an Active Pharmaceutical Ingredient Intermediate Using Inline NIR spectroscopy. Organic Process Research and Development, 16(5), 901-914. https://doi.org/10.1021/op2002563

\section{General rights}

Copyright and moral rights for the publications made accessible in the public portal are retained by the authors and/or other copyright owners and it is a condition of accessing publications that users recognise and abide by the legal requirements associated with these rights.

- Users may download and print one copy of any publication from the public portal for the purpose of private study or research.

- You may not further distribute the material or use it for any profit-making activity or commercial gain

- You may freely distribute the URL identifying the publication in the public portal 


\title{
Monitoring and Control of a Continuous Grignard Reaction for the Synthesis of an Active Pharmaceutical Ingredient Intermediate Using Inline NIR spectroscopy
}

\author{
Albert E. Cervera-Padrell, ${ }^{\dagger}$ Jesper P. Nielsen, ${ }^{\ddagger}$ Michael Jønch Pedersen, ${ }^{\dagger}$ Kim Müller Christensen, ${ }^{\dagger}$ \\ Asmus R. Mortensen, ${ }^{\dagger}$ Tommy Skovby, ${ }^{\ddagger}$ Kim Dam-Johansen, ${ }^{\dagger}$ Søren Kiil, ${ }^{\dagger}$ and Krist V. Gernaey ${ }^{*}, \dagger$ \\ ${ }^{\dagger}$ Department of Chemical and Biochemical Engineering, Technical University of Denmark (DTU), Building 229, DK-2800 Kgs. \\ Lyngby, Denmark \\ ${ }^{\ddagger}$ Chemical Production Development, H. Lundbeck A/S, Oddenvej 182, DK-4500 Nykoebing Sj., Denmark
}

\begin{abstract}
Inline near-infrared (NIR) spectroscopy has been used to monitor a continuous synthesis of an active pharmaceutical ingredient (API) intermediate by a Grignard alkylation reaction. The reaction between a ketone substrate and allylmagnesium chloride may form significant impurities with excess feeding of the Grignard reagent beyond the stoichiometric ratio. On the other hand, limiting this reagent would imply a loss in yield. Therefore, accurate dosing of the two reactants is essential. A feedforward-feedback control loop was conceived in order to maintain the reaction as closely as possible to the stoichiometric ratio, leading the path to full process automation. The feedback control loop relies on NIR transmission measurements performed in a flow cell where, in contrast to labor-intensive offline HPLC analytical methods, the whole reaction product can be scanned in real time without sample dilution. A robust PLS (projection to latent structures) model was developed with a satisfactory standard error of prediction, providing quantification of the ketone substrate in solutions with a high variability of the major solution component-the alkoxide product. In addition, model performance supervision tools such as the spectral residuals or simple plots of pretreated spectra can assist in the identification of spectral outliers, which in this case could be related to Grignard reagent excess. If the sampling time of the NIR instrument is short enough, manipulating the inputs to the reactor may be used to obtain information about its dynamic behavior. This information is very useful for process control design, assessment of analytical tools and definition of sampling times. In this work, a systematic procedure for chemometric model building is followed, after which a discussion is made on some of the potential applications that can be found when exploiting the fast and rich information provided by NIR spectroscopy.
\end{abstract}

\section{INTRODUCTION}

The pharmaceutical industry is making a significant effort to improve productivity, quality, sustainability and flexibility of its manufacturing and research and development (R\&D) activities. ${ }^{1,2}$ Fierce competition and increasing time and cost related to drug discovery and development have motivated a change in the business strategy of pharmaceutical companies, increasingly emphasizing cost reduction and shorter time to market. ${ }^{3,4}$ These objectives must be met through an improvement of the efficiency and sustainability of all processes related to the discovery, development and long-term manufacturing of drug products. Continuous processing (CP), applied throughout all the stages of the life cycle of a drug, ${ }^{3,5}$ provides an opportunity to reduce costs, footprint, energy consumption, solvent utilization and environmental impact while improving quality and control of manufacturing processes and final products. ${ }^{2,4,6-8}$ Indeed, CP was selected as the most significant green engineering research area by the American Chemical Society (ACS) Green Chemistry Institute (GCI) Pharmaceutical Roundtable. ${ }^{2}$ The U.S. Food and Drug Administration (FDA) has also encouraged the adoption of advanced manufacturing and real-time quality-control technologies through the publication of the process analytical technology (PAT) guidance. ${ }^{9}$
Several studies have demonstrated the advantages of performing both active pharmaceutical ingredient (API) synthesis $^{6-8,10,11}$ and drug product formulation ${ }^{1,4,7,12}$ in continuous mode. In the context of organic synthesis-based API production, microreactors $8,10,11,13-15$ and microseparation ${ }^{10,16,17}$ processes have intensively been studied in recent years, providing the basic tools for continuous flow multistep organic synthesis. ${ }^{5,18,19}$ Scaling-down to the microscale results in enhancement of phenomena such as mass transfer and heat transmission via large area-to-volume ratios, while taking advantage of capillarity and surface tension effects at a small scale. $^{8,10,11,14,16,17}$ Smaller process hold-up volumes of potentially toxic and/or dangerous compounds imply safer operation. ${ }^{6,8,11}$ These principles should be conserved across different scales of operation using a scaling-out approach, i.e. replicating small units working in parallel. ${ }^{1,10,14}$ However, fluid distribution and parallelization of microscale units is not such a straightforward task as is often claimed, ${ }^{20}$ and according to Kockmann et al., ${ }^{21}$ it should even be kept as a last option. Furthermore, practical problems such as blockages from solids in suspension still form a main limitation for microreaction

Special Issue: Continuous Processes 2012

Received: September 16, 2011

Published: January 24, 2012 
Scheme 1. Alkylation and hydrolysis reaction

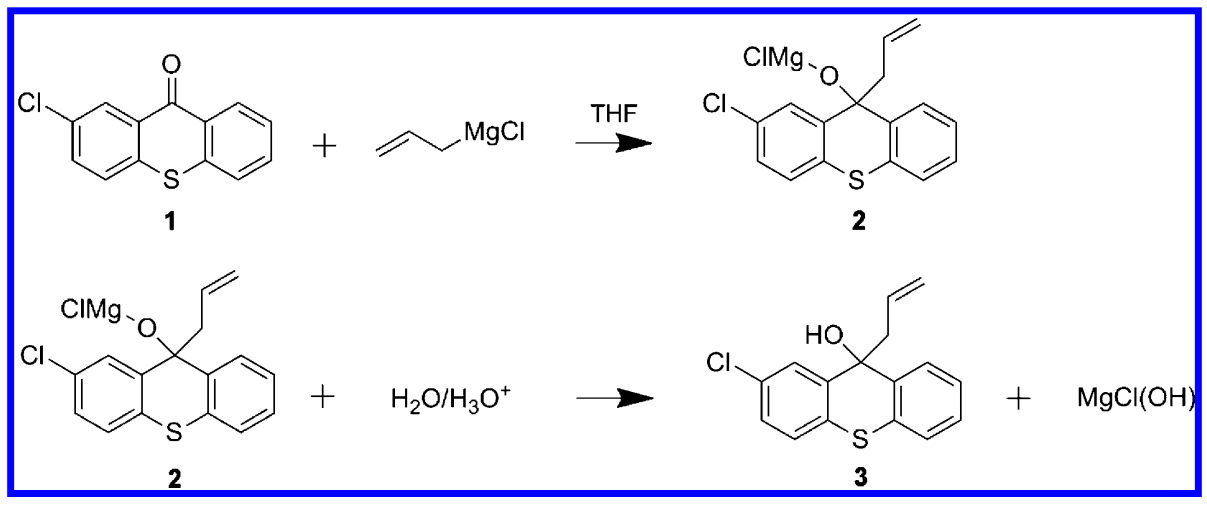

technology. ${ }^{10,22}$ Therefore, a microreactor platform able to scale up while maintaining performance in realistic (challenging) scenarios is highly desired. ${ }^{11,13,21}$ Detailed study of mass and heat transmission characteristics of such a platform should be the basis for the design. ${ }^{13,21}$

Continuous processing is particularly advantageous for fast and exothermic reactions, such as those involving organometallic compounds. ${ }^{11,13}$ These types of reactions (classified as type A according to Roberge et al. $^{23}$ ) have traditionally been carried out in fed-batch mode, slowly dosing one of the reagents in order to prevent a runaway reaction, keeping the reaction mixture under cryogenic temperature (e.g., when high temperature favors side reactions) or alternatively under reflux (e.g., when the reaction heat is very large and is most safely removed exploiting the latent heat of the solvent). ${ }^{11}$ Compounds featuring a low solubility, typically worsened by low temperature, require large solvent amounts if the reaction is to be done in homogeneous conditions. Impurities are commonly formed when $1: 1$ stoichiometry is difficult to accomplish or when hot-spots due to insufficient mixing and heat removal cause side reactions. ${ }^{13}$

Grignard reactions are one of the most common building blocks in organic synthesis. ${ }^{11,24}$ Reactions are extremely rapid and exothermic, and they follow complex reaction mechanisms ${ }^{24}$ which in many cases are not fully understood. While continuous flow reactors may provide improved control over dosing, mixing, monitoring and heat removal, it has been shown that even the use of single-channel microreactors results in high local temperature at the mixing point. ${ }^{13}$ Hot spots can be decreased by following the multi-injection principle, i.e. split up one reagent feed into several streams and thereby also the reaction heat release. ${ }^{13}$ This principle was demonstrated using a Grignard reaction leading to an improvement of the reaction yield. ${ }^{15}$ The multi-injection reactor may also decrease impurity formation when irreversible side reactions are favored by high local concentrations of one of the reagents ${ }^{25}$ (such as in the mixing point).

Continuous-flow reaction systems require online monitoring and feedback control in order to take full advantage of the realtime release PAT concept. ${ }^{9}$ This is particularly important when dealing with Grignard reactions-from a quality control point of view, accurate dosing and keeping the required stoichiometry may contribute to lower impurity formation, and from a risk assessment perspective, fault detection and troubleshooting require timely data. Even though continuous flow reactors are generally claimed to be safer than batch reactors, they introduce other hazards, such as fouling or blockages which can affect a higher number of moving parts (pumps, valves, etc.), potentially resulting in pressure build-up. ${ }^{10,22}$ Monitoring tools should thus provide information about both quality specifications and overall system performance. McMullen and Jensen $^{26}$ review the integration of microreactors with inline physical sensors and analytical chemistry techniques, focusing on reaction monitoring and development of algorithms for automatic reaction screening and optimization. ${ }^{2,28}$ Different ideas for modularization and automation of microreaction technology have been proposed, ${ }^{29}$ showing, for example, simple integration with inline ultraviolet, visible, near-infrared (UVvis-NIR) flow-through cells. In other work, ${ }^{30}$ a microreaction platform was also connected to Raman, NIR, and visible inline spectroscopic techniques to compare their performance for monitoring of a nitration reaction.

NIR spectroscopy is a fast noninvasive analytical method which has found a wide range of applications for the pharmaceutical, agricultural, food and biotechnology industries, among others. ${ }^{31}$ The absorbance bands (overtones or combination bands of absorption bands manifesting in the infrared region of the spectrum) are highly overlapping and often difficult to interpret. However chemometric tools can aid in resolving the spectra. ${ }^{31}$ While IR spectroscopy can, in principle, provide chemical information richer than that of NIR spectroscopy, analytical equipment able to perform inline analysis has only become commercially available recently. ${ }^{32}$ Instrument improvements in NIR spectroscopy, development of fiber optics allowing delocalization of the measurements, and relatively low cost have all contributed to the spread of this analytical technique. ${ }^{31}$ Yet, while the use of NIR or Raman spectroscopy is becoming common practice in laboratory or pilot-scale experiments, ${ }^{26,29,30}$ their application for industrialscale processes under real process conditions (harsh reaction environments, solids in suspension, industrial cleaning processes, etc.) is still a challenge. However, previous work has, for example, demonstrated safer Grignard reagent formation in batch mode through online NIR spectroscopy measurements, allowing real-time monitoring ${ }^{33}$ and control ${ }^{34}$ of the reaction.

In this work, the development of an inline monitoring tool based on NIR spectroscopy for the control of a Grignard alkylation reaction is described. This reaction is the first synthetic step towards the formation of an API, where accurate dosing of the Grignard reagent below or on 1:1 stoichiometry has been found essential to minimize an impurity formation. Monitoring of the ketone substrate in the reaction is especially challenging due to its low solubility compared to that of the product of the alkylation. First, a systematic procedure for chemometric model building with an emphasis on correct 
sampling will be described. Next, the potential of NIR spectroscopy for inline monitoring of this Grignard reaction under real working conditions will be critically evaluated. Finally, a monitoring strategy will be proposed for the reaction under industrial-scale conditions, that is able to show the trend of reactant consumption and identify abnormal situations such as Grignard reagent excess, sudden reagent blockage, or crystallization of the product in the outlet stream. All this information has been found of high interest for safe control of the reaction and opens the path towards process self-regulation and real-time release.

\section{MATERIALS AND METHODS}

2.1. Alkylation Reaction. Allylcarbinol (3, Scheme 1) is the short name for 9-allyl-2-chlorothioxanthen-9-ol, an intermediate product in the production of zuclopenthixol, an API developed by $\mathrm{H}$. Lundbeck A/S. It is produced via a Grignard alkylation reaction of 2-chlorothioxantone (1) (CTX) with allylmagnesium chloride $(\mathrm{Allyl} \mathrm{MgCl})$ in tetrahydrofuran (THF), as shown in Scheme 1. The product of this reaction is an alkoxide (2). The alkoxide is subsequently hydrolyzed in acidic water to produce allylcarbinol and $\mathrm{Mg}$ salts. The continuous hydrolysis of the alkoxide product is explained in detail in a different work. ${ }^{35}$ In this contribution the focus is on the Grignard alkylation reaction, while the alkoxide hydrolysis is only done during sample dilution for HPLC measurement.

A reaction byproduct is formed throughout the reaction in low amount, and increases significantly with an excess of Grignard reagent (AllylMgCl) above the stoichiometric ratio, as shown in Figure 1. It is speculated ${ }^{25}$ that the byproduct

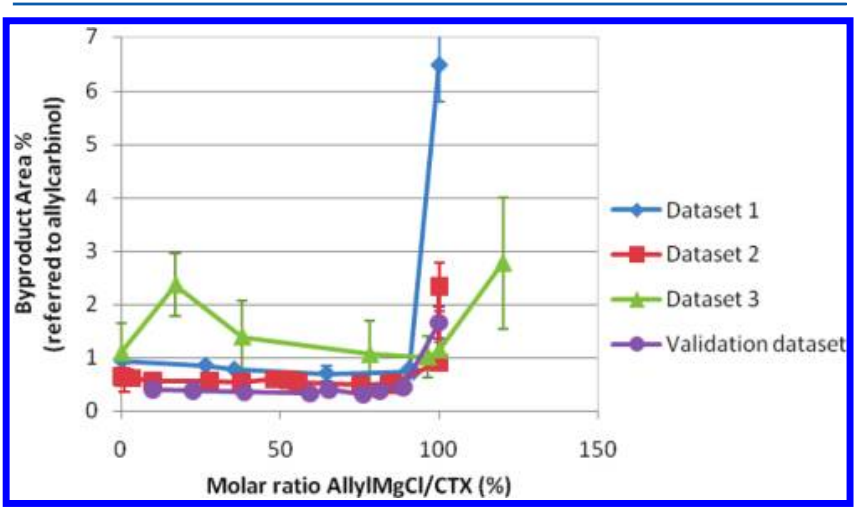

Figure 1. Byproduct peak area \% referred to allylcarbinol peak area (as measured by HPLC, where error bars represent standard deviation for three replicate measurements) as a function of the molar ratio between the Grignard reagent, AllylMgCl, and CTX. Data obtained from the three calibration experiments and the validation experiment are discussed throughout the paper. The data from the third calibration experiment shows a higher impurity formation below the stoichiometric ratio, which is due to a higher Grignard reagent concentration used in the alkylation. The relatively high byproduct formed in calibration experiment 1 above the stoichiometric ratio may be due to higher excess than expected. Absolute peak area values are in any case highest for data set 3 .

formation is caused by reaction of the Grignard reagent with alkoxide product in the absence of CTX and is thus favored by high local concentration of the Grignard reagent. This impurity can be eliminated from the product solution further downstream and it is well-known that it does not pose a product quality risk. However, significant byproduct formation would imply a loss in yield according to the speculated byproduct formation mechanism.

2.2. Continuous Flow Alkylation Reactor. While the alkoxide and allylcarbinol are highly soluble in THF, CTX is sparingly soluble. This physical limitation has led to a design of an alkylation reactor where solvent use has been minimized. ${ }^{25}$ The alkylation reactor is divided into two sections. The first section is a filter reactor (described in a different work ${ }^{25}$ ) that carries out the alkylation of most CTX and yields a highly concentrated alkoxide solution $(\sim 0.7-1 \mathrm{M})$, saturated in CTX $(\sim 0.2-0.3 \mathrm{M})$. The second section of the reactor (Figure 2) must accurately titrate the remaining CTX in solution without exceeding the 1:1 stoichiometry. The reaction is homogeneous and it may be carried out in a tubular reactor, optionally splitting the AllylMgCl flow into several side streams. ${ }^{13,15}$ It is expected that by splitting the Grignard reagent flow into several streams the local concentrations at the mixing point will be lower, resulting in lower impurity formation. ${ }^{25}$ This is thus a different argument in favor of using the multi-injection principle, compared to the one given by Barthe et al., ${ }^{13}$ who focus on heat removal and high local temperatures at the mixing point.

The reactor setup is shown in Figure 2. Up to three side streams could be used to split the Grignard reagent flow into the tubular reactor. Mixing between the alkoxide solution saturated in CTX originating from the filter reactor and the Grignard reagent occurred in 3/16 in., 27 elements Koflo static mixers (SM-1, SM-2, and SM-3). The mixers were connected to around $50 \mathrm{~cm}$ of $1 / 8 \mathrm{in}$. OD stainless steel coiled tube (Swagelok) that was used to complete heat removal and provide an extra residence time. The reaction rate was extremely fast and mixing controlled. The filter reactor was operated at $20{ }^{\circ} \mathrm{C}$ (below room temperature), and the sideentry tubular reactor was immersed into a bath at $30{ }^{\circ} \mathrm{C}$. The bath was set at a higher temperature than the filter reactor in order to avoid precipitation of the CTX solution (saturated at $20{ }^{\circ} \mathrm{C}$ ) which could otherwise cause a blockage. It was assumed that the reaction heat was low enough to be absorbed by the specific heat of the solution (initially at $20^{\circ} \mathrm{C}$ ) or by the bath at $30{ }^{\circ} \mathrm{C}$. Previous experiments have shown that reaction temperature had no effect on impurity formation below 45 ${ }^{\circ} \mathrm{C}$ and thus a precise control of the reaction temperature was not a priority, but rather the reagent stoichiometry and keeping the solution homogeneous. Pumping of the CTX-saturated alkoxide solution was done using an Omega PHP-212B-T diaphragm pump (P-2), after which a check valve (CV-1) was installed (Swagelok, $1 / 8$ in., 25 psi cracking pressure) in order to avoid backflow. Pumping of the AllylMgCl was done using a peristaltic pump (P-1) equipped with a PTFE tube (Masterflex 07523-90 driver equipped with 77390-00 PTFE tubing pump head). Each side entry to the tube reactor was equipped with a check valve (CV-2, CV-3, and CV-4, Swagelok, 1/8 in., 25 psi cracking pressure) to avoid any backflow and equalize pressure drop through each side entry, thereby equilibrating flow rate through the side entries (the check valve cracking pressure was much higher than the liquid pressure drop in the tubular reactor and static mixers; therefore, differences in pressure drop between the different entries could be neglected). It was critical to avoid any contact of the Grignard reagent solution with air (they would otherwise react, forming insoluble $\mathrm{Mg}$ salts), and therefore any solvents used to flush the reactor, the Grignard reagent, the alkoxide solution saturated in CTX, and the product alkoxide solution with titrated CTX were all covered 


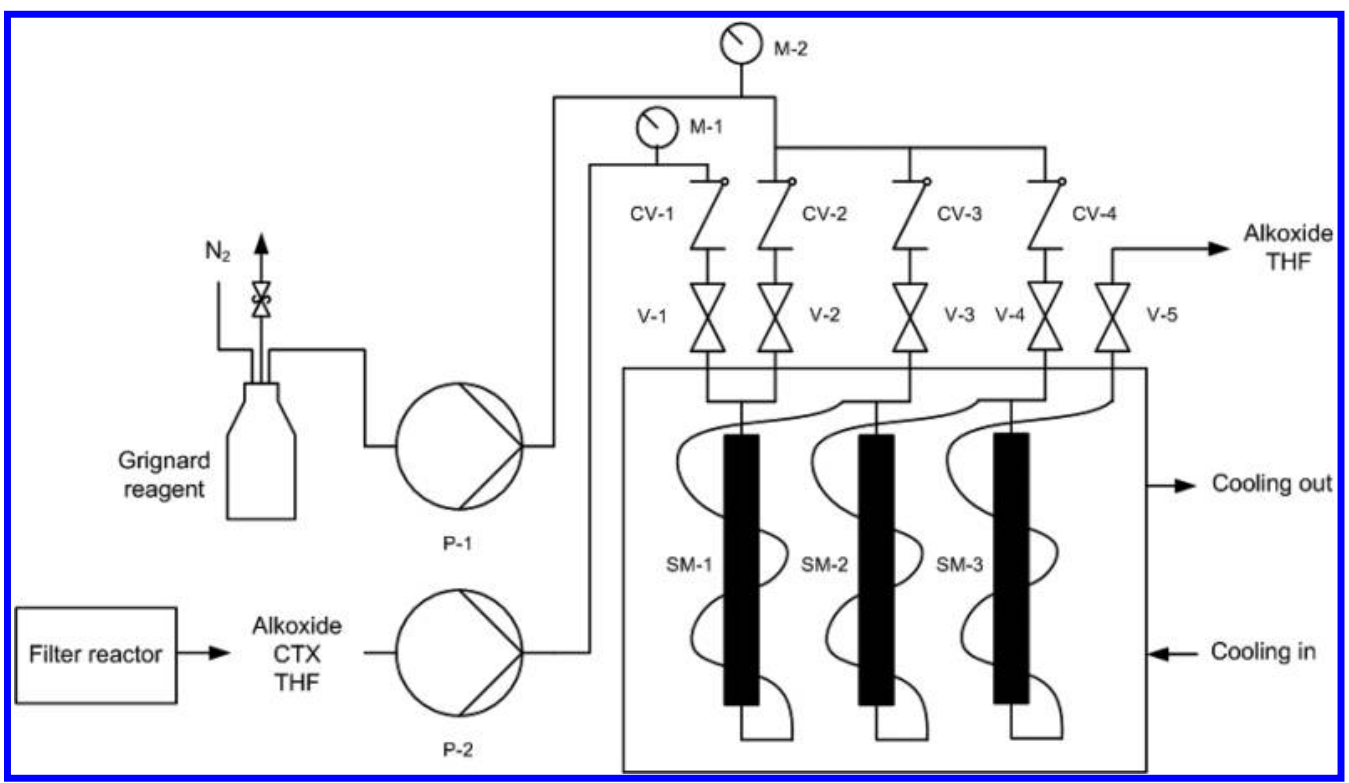

Figure 2. Detail of the second section of the alkylation reactor used for the continuous synthesis of the API intermediate allylcarbinol.

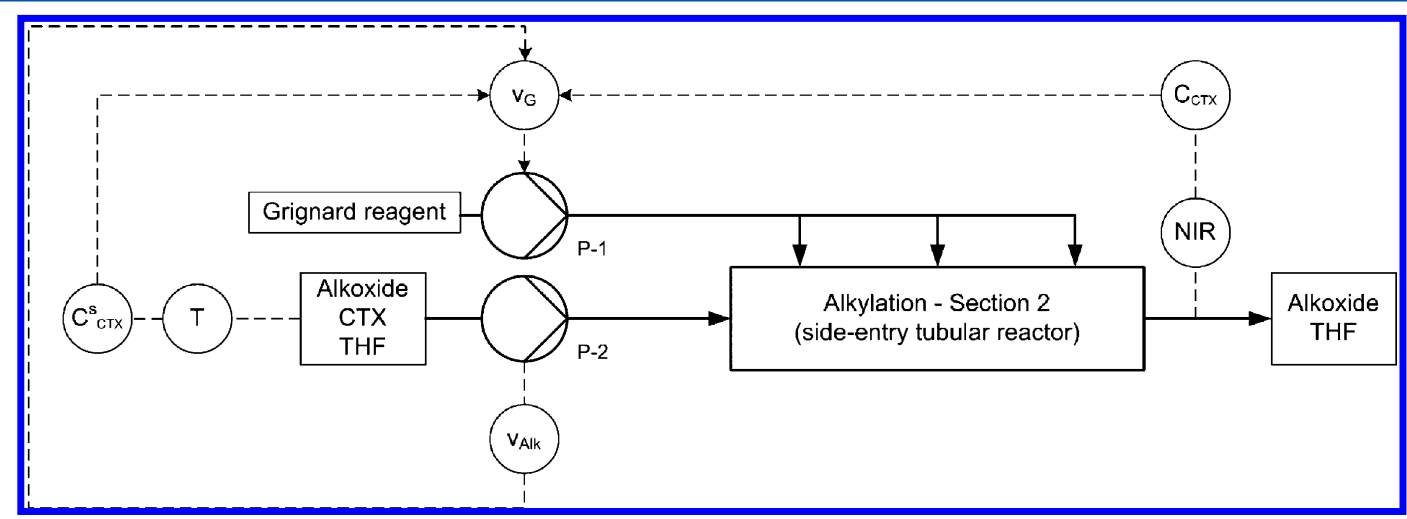

Figure 3. Control strategy diagram. $T$ is the temperature, $C_{\mathrm{CTX}}^{\mathrm{s}}$ is the CTX concentration at saturation for temperature $T$, $v_{\mathrm{G}}$ is the Grignard reagent flow rate, $v_{\mathrm{Alk}}$ is the alkoxide solution flow rate from the filter reactor, and $C_{\mathrm{CTX}}$ is the CTX concentration in the outlet stream.

with nitrogen. Experience demonstrated that only pumps where the liquid is completely isolated from the moving mechanism of the pump were suitable, with the list of possibilities practically reduced to diaphragm pumps and peristaltic pumps. Due to the high THF solvent strength, wet materials had to be either PTFE/PFE/PFA-based or stainless steel. Since fluoropolymers have a low (but still important) oxygen and moisture permeability, flexible tube connections were only used where required for practical reasons. While this solution is acceptable for very short run time in a laboratory setting, a pilot-plant or an industrial-scale reactor should basically use stainless steel material or other compatible metals.

2.3. Control Strategy. The alkylation reactor should preferably be self-regulated, which requires proper monitoring and control. The product from the filter reactor (first section of the alkylation reactor) can be kept saturated in CTX by regulating the Grignard reagent feed rate so that it is slower than the CTX dissolution rate (see ref 25). Concomitantly, the CTX solid feed rate to the filter reactor can be regulated on the basis of online turbidity measurements in the filter reactor, ensuring that there is a constant suspension of CTX. Therefore, under design conditions it is expected that an alkoxide rich, CTX-saturated solution enters the second section of the alkylation reactor (tubular reactor), in which the CTX concentration at saturation can be related to the temperature in the filter reactor. In the second section of the alkylation reactor, it was concluded that the optimal control structure would be feedforward-feedback controlled (Figure 3).The feedforward control loop should measure: (a) the flow rate of CTX-rich alkoxide solution from the first section of the alkylation reactor (filter reactor); (b) the temperature in the filter reactor, in order to calculate the predicted CTX concentration at saturation (according to a predetermined CTX solubility curve in THF containing alkoxide); and (c) it should retrieve from a database the Grignard reagent concentration in the storage tank, or alternatively measure it in real time. With this information it would be simple to calculate the flow rate of Grignard reagent required for 95$100 \%$ CTX titration, since the controller objective would be to maintain the Grignard reagent stoichiometry on (or a little below) $1: 1{ }^{a}$ However, measurement errors and/or delays and solubility model inaccuracies could lead to incorrect titration. Furthermore, unknown disturbance rejection and troubleshooting are only possible by measuring the product concentration in the outlet stream and including a feedback control loop. If the measurement error is low enough, measuring the outlet streamflow rate and the alkoxide concentration could facilitate real time release and would 
provide the information needed to calculate how much operation time would be required to collect a batch. In this work, product monitoring in the outlet stream was carried out using inline NIR spectroscopy measurements.

2.4. Chemometric NIR Model Development. The general procedure for chemometric model building is shown as a diagram in Figure 4. First, absorption spectra of pure

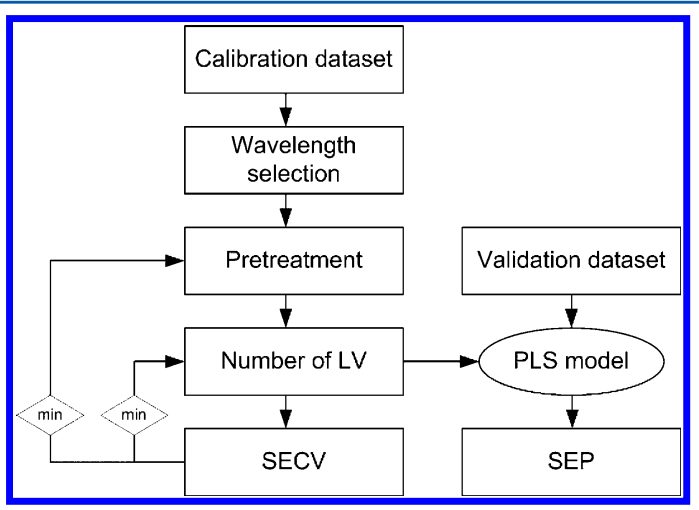

Figure 4. Procedure for chemometric model building. Note that only aiming at minimizing the standard error of cross-validation (SECV) may lead to overfitting. Therefore, both the number of LV and SECV were minimized. Overfitting was detected with an independent validation and quantified by the standard error of prediction (SEP).

compounds were inspected in order to select relevant wavelength intervals for chemometric model development. The wavelength intervals were compared with literature data to relate absorption peaks with chemical structure information. Next, different mathematical pretreatments ${ }^{31}$ were applied to the raw absorption spectra in order to eliminate baseline and/ or first order shifts caused by scattering, instrument noise, bending of fiber optic cables, etc. Then, a projection to latent structures (PLS, also known as partial least-squares) regression model ${ }^{36}$ was built based on a calibration data set. Leave-one-out cross-validation ${ }^{37}$ was used to generate a standard error of cross-validation (SECV) plot, from which the minimum number of significant latent variables was selected to avoid model overfitting. The SECV was compared for the different pretreatments tested and the best model was validated against an independent validation data set, obtaining a standard error of prediction (SEP). The SECV and SEP were calculated in an analogous way (eq 1 , where $C_{i}^{\mathrm{NIR}}$ is the concentration of an analyte in sample $i$ as predicted by the PLS model using the NIR spectra, $C_{i}^{\mathrm{HPLC}}$ is the concentration of the same analyte as given by the reference analytical method, such as HPLC, and $n$ is the number of samples).

$$
\mathrm{SECV} / \mathrm{SEP}=\sqrt{\frac{\sum_{i=1}^{n}\left(C_{i}^{\mathrm{NIR}}-C_{i}^{\mathrm{HPLC}}\right)^{2}}{n}}
$$

2.4.1. Atline Determination of Grignard Reagent Concentration in a Stock Solution. Grignard reagent strength diminishes with time due to for example exposure of open bottles to moisture or air, even when extreme care is taken to keep solutions under nitrogen cover. Therefore, it is required to analyze the reagent strength before use. The recommended analysis method is a potentiometric titration using 2-butanol ${ }^{24}$ under nitrogen cover, which is expensive, complex and timeconsuming to carry out and requires considerable human expertise. It is much simpler and faster to build a chemometric model based on atline NIR spectroscopy measurements, obtaining a Grignard concentration value within seconds. A PLS model was built by opening a new bottle of $2 \mathrm{M}$ Grignard reagent from Sigma Aldrich and making dilutions with THF under nitrogen cover, immediately scanning each diluted sample. The prediction ability of the model was evaluated in terms of SECV $(0.06 \mathrm{M})$. Even though this is only a rough model and using the SECV to evaluate prediction ability is certainly optimistic, this procedure was considered acceptable for the purpose of this model (obtain a rough estimate of the Grignard strength).

2.4.2. Inline determination of CTX concentration. A preliminary experiment was carried out to determine the spectral changes observed during the alkylation of a solution of CTX in THF (not containing alkoxide initially). THF was used as reference spectrum for calculation of absorption spectra. CTX was dissolved in THF close to saturation and was reacted with Grignard reagent using an old version of the tubular reactor depicted in Figure 2. The flow rate of Grignard reagent was varied from 0 up to the stoichiometric value for full CTX conversion. After each flow rate change the reactor was kept running at fixed flow rates for $5 \mathrm{~min}$ in order to reach steady state.

Once the important wavelength intervals were visually identified, 3 calibration experiments and 1 validation experiment were performed. All NIR measurements used THF as reference. The 3 calibration experiments were carried out following a similar procedure. An alkoxide-rich CTX-saturated solution was obtained using a filter reactor. ${ }^{25}$ The solution was then pumped through the second section of the alkylation reactor (Figure 2, side-entry tubular reactor) at a fixed flow rate of $10 \mathrm{~mL} / \mathrm{min}$, changing the flow rate of Grignard reagent from 0 to the stoichiometric point and if possible beyond. Unfortunately, for calibrations 1 and 2, the stock solution of alkoxide-rich CTX-saturated solution ran out when the stoichiometric point was reached. After each change of flow rate the reactor was kept running using fixed flow rates for 5 min in order to reach steady state, and sampling was performed as described in section 2.6. The difference between the 3 calibration experiments was the initial alkoxide concentration (135, 225, and $290 \mathrm{~g} / \mathrm{L})$ and the Grignard strength used (0.6, 1.1 , and $2 \mathrm{M}$, respectively). This calibration procedure was followed with the purpose of (a) eliminating correlation between the change in concentration of the two analytes and thereby obtain PLS models specific to each analyte; ${ }^{38}$ (b) obtaining a robust PLS model for CTX, including variability in alkoxide concentration around the expected set point ( $215 \mathrm{~g} /$ $\mathrm{L})$; and (c) generating a very rough alkoxide PLS model.

The validation experiment was run similarly to the calibration experiments. The initial alkoxide concentration (solution from the filter reactor) was $180 \mathrm{~g} / \mathrm{L}$. This solution was pumped through the tubular reactor at a fixed flow rate of $10 \mathrm{~mL} / \mathrm{min}$, changing the flow rate of Grignard reagent according to the profile outlined in Figure 10. The flow rate was adjusted to test the prediction ability against sudden changes of Grignard reagent and also against smooth changes around the stoichiometric point (full titration of the CTX). After each flow rate change, the conditions were fixed for $5 \mathrm{~min}$ in order to reach steady state.

2.5. Analytical Instrument and Software. An FT-NIR process analyzer (Networkir, Q-Interline/ABB) equipped with an InGaAs detector measuring in the range $800-2600 \mathrm{~nm}$ was connected to a CUV-UV holder for atline transmission 


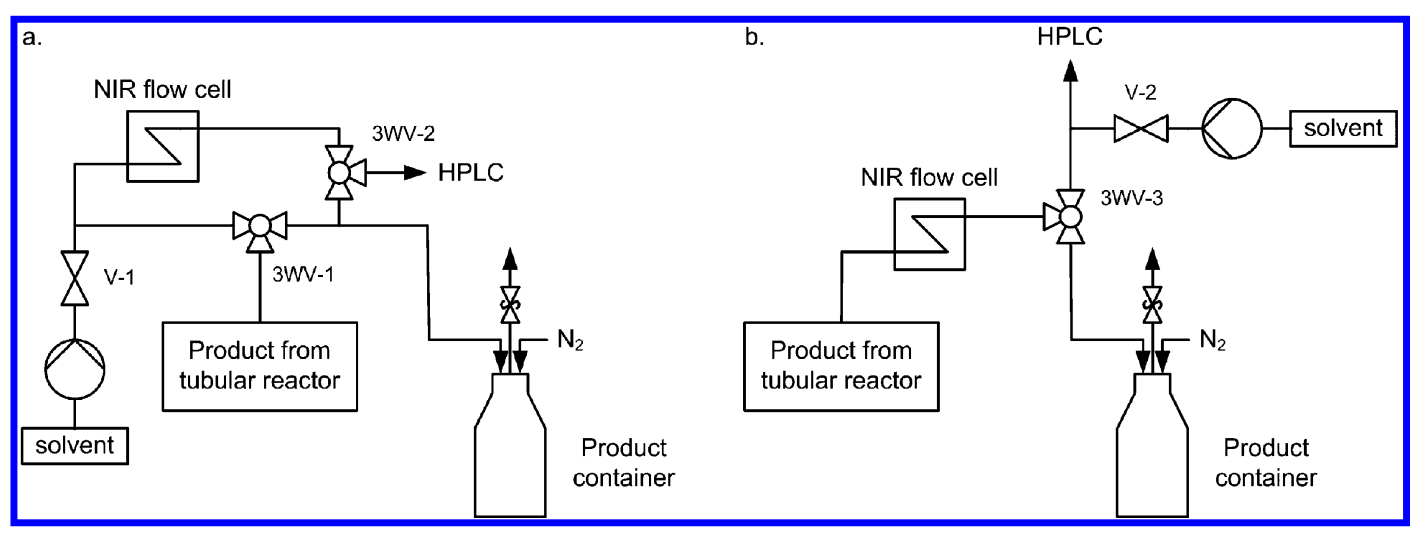

Figure 5. Sampling setups used for calibration (a) and validation (b) of the chemometric model used to determine CTX concentration in the alkoxide solution.

measurements in $1 \mathrm{~cm}$ cuvettes (OceanOptics), optimized for the range $200-2000 \mathrm{~nm}$, using two $500 \mu \mathrm{m}$ fiber optic cables. A homemade Teflon vial holder for 8-mm path length disposable borosilicate glass vials (Kimble) was inserted into the CUV-UV holder, enabling very fast measurements with disposable vials. Atline measurements of the Grignard reagent were taken at ambient temperature (not active control). Sixteen scans were averaged for each sample, and the resolution was set to 16 $\mathrm{cm}^{-1}$. A second InGaAs detector measuring in the range 800$2100 \mathrm{~nm}$ was connected to a FIA-Z-SMA-TEF flow cell (OceanOptics) for inline transmission measurements $(1 \mathrm{~cm}$ path length), optimized for the range $210-2000 \mathrm{~nm}$, using two $15 \mathrm{~m}$ long $300 \mu \mathrm{m}$ fiber-optic cables in order to reach a fume hood containing the reactor setup. Sixteen scans were averaged for each sample, and the resolution was set to $16 \mathrm{~cm}^{-1}$. With these settings the sampling time was $5 \mathrm{~s}$. The inner volume of the flow cell was only $50 \mu \mathrm{L}$, which was critical for correct sampling. The product from the tubular reactor was connected to the flow cell using $1 / 4-28$ Upchurch fittings and $1 / 8$ in. OD PFA tubing (Swagelok). Spectra were acquired and processed using GRAMS/AI 7.0 (Thermo Electron Corporation). Chemometric models were built using the add-on PLSplus/ IQ (Thermo Electron Corporation). Plots were generated with MATLAB.

2.6. Sampling Setup. NIR spectroscopy is usually treated as a secondary analytical technique, meaning that it must be calibrated against a reference analytical technique, in this case HPLC. Correct sampling avoiding cross-contamination in calibration experiments built from inline NIR measurements is not straightforward, and must be taken into account in the assembly of the sampling setup. Solids precipitation on the tube walls could for example induce experimental errors. Correct synchronization of the NIR and the reference analysis method and ensuring steady-state conditions before analysis are also very important. Two sampling setups were assembled. The first setup (Figure 5a) was used for calibration, where NIR measurements were taken discontinuously and both the NIR flow cell and the HPLC sampling tube were flushed before each analysis. The second setup (Figure $5 b$ ) was used for validation, where NIR measurements were taken continuously, and thus only the HPLC sampling tube was flushed before sampling. In the first setup (Figure 5a), the product from the tubular reactor was normally directed to a product container, until steady state was reached. At that point (usually $4 \mathrm{~min}$ after a flow rate change), the liquid was sent to the NIR flow cell using the three-way valve $3 \mathrm{WV}-1$, with $\mathrm{V}-1$ closed. The flow cell was flushed for about $30-45 \mathrm{~s}$ with the product at steady-state conditions opening the outlet $3 \mathrm{WV}-2$ for HPLC sampling, and then a single NIR measurement (16 coadded scans) was taken while collecting a small sample for HPLC analysis. Next, the three-way valve $3 \mathrm{WV}-1$ was turned again to send the product to the product container, and the Grignard reagent flow rate was changed in order to generate the next product concentration. While waiting for steady state, fresh solvent (THF) was flushed (opening V-1) through the NIR flow cell and the HPLC sampling tube in order to prevent cross-contamination.

In the second setup (Figure $5 b$ ), the product from the tubular reactor was continuously scanned by the NIR flow cell (16 co-added scans for each measurement, every $10 \mathrm{~s}$ ). The liquid at the outlet of the flow cell could either be sent to a product container or for HPLC reference sampling. The setup was analogously operated, and the HPLC sampling tube was properly flushed with THF after each sample.

2.7. HPLC Reference Analysis. The concentrations of the alkoxide, CTX, and impurities were determined using HPLC analysis. A LaChrome Elite HPLC machine equipped with a Phenomenex Gemini C6-Phenyl column for reverse phase HPLC using a gradient method based on acetonitrile and aqueous buffer (ammonium formate $\mathrm{pH}$ 9) as mobile phases were employed for the analysis of previously diluted samples. Each $50 \mu \mathrm{L}$ sample was diluted with $4.95 \mathrm{~mL}$ of THF. Then 50 $\mu \mathrm{L}$ of these solutions was further diluted with $950 \mu \mathrm{L}$ of mobile phase at time 0 of the gradient method (total dilution factor 2000). Samples were taken in triplicates, since repeatability of the dilution procedure was the major source of experimental error. Calibration curves were built from allylcarbinol and CTX standards, obtaining very satisfactory regression coefficients.

\section{RESULTS AND DISCUSSION}

3.1. Chemometric Model Development. 3.1.1. Overview of Absorption Spectra-Wavelength Interval Selection. The three analytes involved in the alkylation reaction (CTX, AllylMgCl, and alkoxide) have a significant part of their chemical structures in common, and thus their absorption bands in the NIR spectrum are highly overlapping (see for example Figure 6). Basically, AllylMgCl transfers an allyl group to a molecule containing two aromatic groups (CTX), transforming a ketone into an alkoxide. The ketone (which is the only chemical group unique for CTX and would thus be a very good indicator for the disappearance of this molecule) does only manifest in the NIR spectrum as a very weak stretch second overtone $(1900-2050 \mathrm{~nm})^{39}$ that could not be detected 


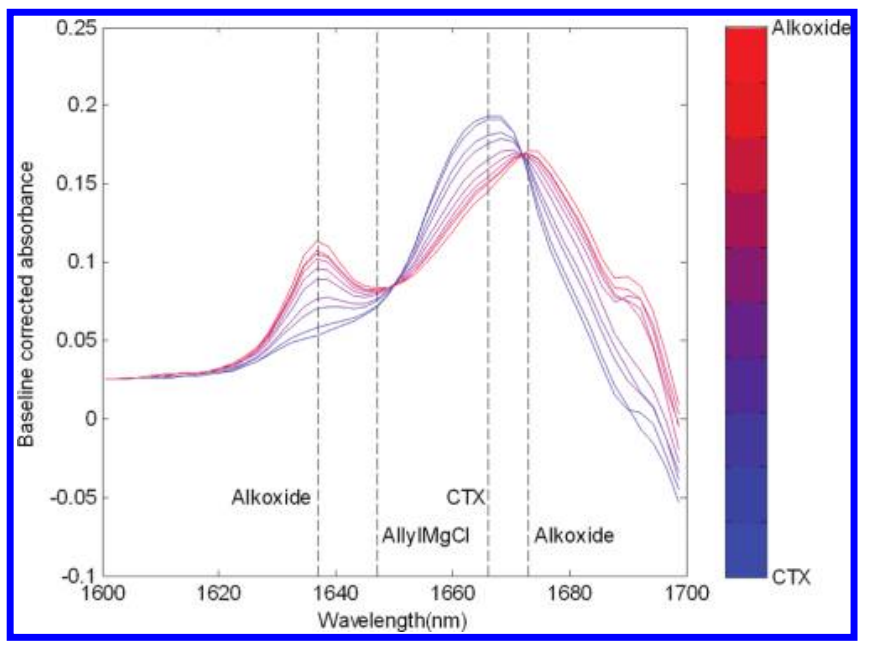

Figure 6. Baseline corrected spectra obtained during the alkylation of a CTX-saturated solution in THF. The color changes from blue to red proportionally to the CTX conversion. The peak maxima for each analyte are plotted as dashed lines.

in practice. In contrast, the $\mathrm{C}-\mathrm{H}$ stretch first overtone of the aromatic rings (around $1685 \mathrm{~nm})^{39}$ and the allyl group (around $1620 \mathrm{~nm})^{39}$ have clear absorption bands. The solvent THF absorbs in two bands around 1688 and $1754 \mathrm{~nm}$, corresponding to the $\mathrm{C}-\mathrm{H}$ stretch first overtone of the $\mathrm{CH}_{2}$ groups. ${ }^{39}$ Since THF is used as a reference spectrum, the wavelength regions where THF absorbs are highly noisy and thus cannot be used to measure the analytes. The spectra acquired during the alkylation of a saturated solution of CTX in THF (not containing alkoxide) are plotted in Figure 6. The formation of alkoxide from the CTX solution is observed as the growth of the allyl absorption band at $1637 \mathrm{~nm}$. The disappearance of CTX can be observed as a slight decrease in the aromatic absorption band at $1666 \mathrm{~nm}$ combined with a slight shift towards longer wavelengths (peak at $1673 \mathrm{~nm}$ ). Spectra obtained from high concentration alkoxide solution (such as those measured in calibrations $1-3$ and in the validation experiment) show much higher bands (allyl group and aromatic group) corresponding to the alkoxide, but still a small shift in the aromatic absorption band can be observed when CTX is depleted. The aromatic absorption band is very near to the noisy area caused by high THF absorption. This spectrastructure relation information guided the selection of wavelength intervals for chemometric model development.

3.1.2. PLS Model Calibration and Cross-Validation. Several PLS models were constructed on the basis of a data set including the three calibration alkylation reactions, with initial alkoxide concentrations around 135, 225, and $290 \mathrm{~g} / \mathrm{L}$ for the first, second, and third reactions, respectively (Table 1). In all

Table 1. Initial alkoxide concentrations (product from the filter reactor) obtained in the three calibration experiments and the validation experiment ${ }^{a}$

\begin{tabular}{cccccc} 
& \multicolumn{3}{c}{ calibration data set } & \multicolumn{1}{c}{$\begin{array}{c}\text { validation } \\
\text { data set }\end{array}$} \\
\cline { 2 - 4 } $\begin{array}{c}\text { initial alkoxide } \\
\text { concentration }\end{array}$ & $135 \mathrm{~g} / \mathrm{L}$ & $225 \mathrm{~g} / \mathrm{L}$ & $290 \mathrm{~g} / \mathrm{L}$ & $180 \mathrm{~g} / \mathrm{L}$
\end{tabular}

${ }^{a_{T}}$ The three calibration data sets were combined into a single calibration. experiments the CTX concentration was varied from $0 \mathrm{~g} / \mathrm{L}$ up to close to the saturation point $(\sim 50-55 \mathrm{~g} / \mathrm{L})$. The PLS models reported in Table 2 were obtained by following the procedure outlined in Figure 4. If necessary, the wavelength interval was narrowed slightly to avoid noise introduced by the Savitzky-Golay ${ }^{31,37}$ pretreatments in the neighborhood of the THF absorption band. Five different pretreatments ${ }^{31,37}$ were tested: two-point baseline correction (BC) based on an area without analyte absorption, first derivative (Savitzky-Golay), second derivative (Savitzky-Golay), ${ }^{31,37}$ multiplicative scatter correction (MSC) and standard normal variate (SNV). In addition, mean-centering (MC) and/or variance scale (VS) were used to try to increase the sensitivity of the models to the small variations in the spectra given by the low CTX concentrations. Different numbers of smoothing points were tested $(7,11,15)$ for the Savitzky-Golay derivatives, while 11 points was considered the right number for the resolution used. Some combinations of the above-mentioned pretreatments were also tested (e.g., derivative $+\mathrm{BC}$ ), although large changes were not expected since the derivatives compensate for bias (first derivative) and slope (second derivative) shifts (note that models 10 and 12 in Table 2 give exactly the same result).

The number of latent variables (LV) was selected on the basis of SECV vs LV plots. While it should be expected to obtain a minimum between model underfit and overfit, ${ }^{31,37}$ the number of LV for this minimum was in many situations too large, with the model tending to overfit the calibration data (as shown by poor prediction of independent validation samples). Therefore, the right number of LV was selected by visual inspection, increasing the number of LV until large decreases of the SECV were not longer observed. BC and first and second derivatives gave a similar result in cross-validation prediction ability (SECV between 4 and $4.5 \mathrm{~g} / \mathrm{L}$ ), while MSC and SNV clearly underperformed for this particular application. Model 5 was selected on purpose with a high number of LV (6 was the $\mathrm{LV}$ at the minimum SECV) in order to show overfitting (SECV $=2.7 \mathrm{~g} / \mathrm{L}$, however SEP $=30 \mathrm{~g} / \mathrm{L}$ ).

Figure 7 shows the cross-validation prediction values given by the best model found (model 10) for the three data sets used for calibration (initial alkoxide concentrations 135, 225, and $290 \mathrm{~g} / \mathrm{L}$ corresponding to data sets 1,2 , and 3 in the figure, respectively). The NIR cross-validation predictions lie relatively near to the $y=x$ line, following the trend of CTX concentrations determined by the reference HPLC measurements. The horizontal error bars represent the repeatability of each HPLC measurement, calculated as the standard deviation for three replicate measurements. The vertical error bars represent the SECV of the PLS model. The extreme values (high and low ends of CTX concentration) are especially difficult to predict since the model may need to extrapolate. The repeatability of the HPLC measurements was especially low (high standard deviation for the replicate measurements) for the data set 2, especially for the point with highest concentration. It has been confirmed that this large measurement error is introduced by the dilution procedure required for HPLC analysis (dilution factor 2000), since the variations in concentration between replicates for the two analytes measured by HPLC (CTX and alkoxide) are highly correlated. Small differences in pipet tips (for example they behave differently when they are wet and when they are dry) and the high volatility of the solvent (THF) affect the sample volume. Data set 2 was actually the first experiment carried out. Extreme care was taken afterwards in order to try to improve the repeatability 
Table 2. PLS models developed for the quantification of CTX in an alkoxide solution ${ }^{a}$

\begin{tabular}{|c|c|c|c|c|c|c|c|c|c|c|c|}
\hline \multirow[b]{2}{*}{ model } & \multirow[b]{2}{*}{ MC/VS } & \multirow[b]{2}{*}{ pretreatment } & \multirow[b]{2}{*}{ wavelength $(\mathrm{nm})$} & \multicolumn{3}{|c|}{ cross-validation } & \multicolumn{5}{|c|}{ validation } \\
\hline & & & & LV & SECV (g/L) & $Q^{2}$ & SEP $(g / L)$ & $Q^{2}$ & bias & slope & SEP corr $(g / L)$ \\
\hline 1 & $\mathrm{MC}$ & $\mathrm{BC}$ & $1610-1684$ & 3 & 4.5 & 0.95 & 7.7 & 0.99 & 3.53 & 1.14 & 1.4 \\
\hline 2 & MC-VS & $\mathrm{BC}$ & $1610-1684$ & 2 & 4.6 & 0.95 & 4.5 & 0.99 & -1.12 & 1.17 & 1.4 \\
\hline 3 & - & $1 \mathrm{der}, 11 \mathrm{pts}$ & $1610-1673$ & 2 & 4.5 & 0.95 & 5.2 & 0.99 & 2.18 & 1.10 & 1.4 \\
\hline 4 & $\mathrm{MC}$ & $1 \mathrm{der}, 11 \mathrm{pts}$ & $1610-1673$ & 2 & 4.4 & 0.95 & 5.0 & 0.99 & 0.86 & 1.13 & 1.4 \\
\hline 5 & MC-VS & $1 \mathrm{der}, 11 \mathrm{pts}$ & $1610-1673$ & 6 & 2.7 & 0.98 & 30.3 & 0.99 & 26.58 & 1.14 & 1.6 \\
\hline 6 & $\mathrm{MC}$ & 1 der, BC & $1610-1673$ & 2 & 4.2 & 0.95 & 4.9 & 0.99 & 0.70 & 1.13 & 1.4 \\
\hline 7 & $\mathrm{MC}$ & $1 \mathrm{der}, 15 \mathrm{pts}$ & $1610-1673$ & 3 & 3.5 & 0.97 & 4.7 & 0.99 & 0.75 & 1.12 & 1.6 \\
\hline 8 & $\mathrm{MC}$ & $1 \mathrm{der}, 7 \mathrm{pts}$ & $1610-1673$ & 2 & 4.4 & 0.95 & 4.8 & 0.99 & 0.77 & 1.13 & 1.4 \\
\hline 9 & - & 2 der, 11pts & $1610-1673$ & 2 & 4.9 & 0.94 & 4.9 & 0.99 & 2.08 & 1.09 & 1.4 \\
\hline 10 & MC & 2der, 11pts & $1610-1673$ & 3 & 4.2 & 0.95 & 3.2 & 0.99 & -0.52 & 1.11 & 1.4 \\
\hline 11 & MC-VS & $2 \mathrm{der}, 11 \mathrm{pts}$ & $1610-1673$ & 4 & 4.0 & 0.96 & 5.3 & 0.99 & -7.95 & 1.14 & 1.4 \\
\hline 12 & $\mathrm{MC}$ & 2der, BC & $1610-1673$ & 3 & 4.2 & 0.95 & 3.2 & 0.99 & -0.52 & 1.11 & 1.4 \\
\hline 13 & $\mathrm{MC}$ & 2 der, $15 p t s$ & $1610-1673$ & 4 & 4.1 & 0.96 & 3.6 & 0.99 & 0.15 & 1.10 & 1.7 \\
\hline 14 & $\mathrm{MC}$ & $2 \mathrm{der}, 7 \mathrm{pts}$ & $1610-1673$ & 2 & 4.6 & 0.94 & 5.1 & 0.99 & 0.90 & 1.13 & 1.4 \\
\hline 15 & $\mathrm{MC}$ & MSC & $1610-1684$ & 5 & 6.8 & 0.88 & 5 & 0.98 & 3.33 & 1.04 & 2.5 \\
\hline 16 & - & SNV & $1610-1684$ & 3 & 6.9 & 0.88 & 10.9 & 0.98 & -1.56 & 0.69 & 2.5 \\
\hline 17 & - & SNV detrend ${ }^{31,37}$ & $1610-1684$ & 3 & 6.8 & 0.88 & 8.8 & 0.99 & 14.69 & 0.67 & 1.7 \\
\hline 18 & $\mathrm{MC}$ & SNV & $1610-1684$ & 2 & 7.7 & 0.85 & 10.9 & 0.98 & -1.60 & 0.69 & 2.4 \\
\hline 19 & $\mathrm{MC}$ & SNV detrend & $1610-1684$ & 3 & 6.4 & 0.89 & 7.5 & 0.99 & 12.77 & 0.69 & 1.7 \\
\hline 20 & MC-VS & SNV detrend & $1610-1684$ & 3 & 7.1 & 0.87 & 7 & 0.99 & 11.90 & 0.70 & 1.9 \\
\hline
\end{tabular}

${ }^{a}$ MC: Mean-Centering, VS: Variance Scale, BC: Baseline correction, 1der: First Derivative (Savitzky-Golay), pts: smoothing points, 2 der: Second Derivative (Savitzky-Golay), MSC: Multiplicative Scatter correction, SNV: Standard Normal Variate, LV: number of Latent Variables, SECV: Standard Error of Cross-Validation, $Q^{2}$ : cross-validation/validation regression coefficient, SEP: Standard Error of Prediction, SEP corr: SEP corrected for bias and slope deviation.

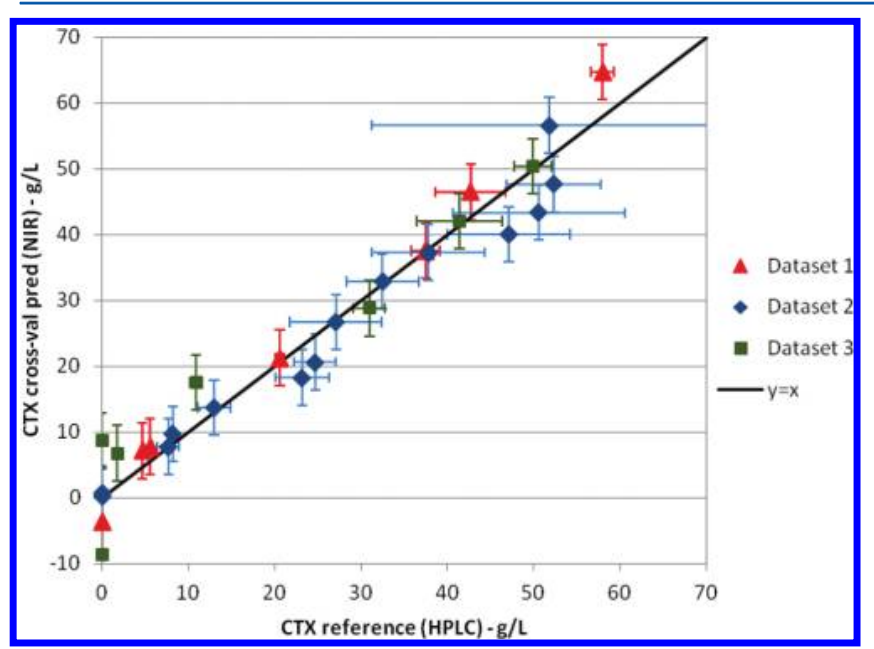

Figure 7. Leave-one-out cross-validation prediction values vs HPLC reference values for data sets 1,2 , and 3 (with initial alkoxide concentrations of 135,225 , and $290 \mathrm{~g} / \mathrm{L}$, respectively).

as much as possible. Data sets 1 and 3 resulted in lower standard deviations between replicates. Despite the high measurement errors introduced in some experimental points, no points were discarded as concentration outliers. On the one hand, taking the average of three replicate measurements for each point reduces the influence of measurement errors. On the other hand, it was considered positive to conserve as much variability as possible in the calibration data set, in order to include several high concentration values, as well as to provide a realistic quality check for the model (the SECV provides a very good estimate of the prediction ability of the PLS model and will probably tend to overfit less). Furthermore, the figure alerts that using offline HPLC samples during production could involve significant measurement biases leading to the wrong control decisions, while real-time inline NIR data (without sample dilution) should be less prone to measurement biases if it is combined with the right model supervision tools. The lowest predicted concentration value (corresponding to data set 3) was actually identified as a spectrum outlier (containing excess of Grignard reagent as discussed below), and could thus be removed from the calibration.

The structure of the PLS model 10 was examined by plotting the scores of the latent variable 1 (LV1) against the scores of LV2 (Figure 8a), the loadings of LV1, LV2, and LV3 (Figure $8 \mathrm{~b}$ ) and relating them to the mean-centered second derivative of the spectra in the calibration data sets (Figure 8c). The scores plot (Figure 8a) shows that the three data sets used for calibration are clearly separated as three populations. The scores of LV1 are correlated with the CTX concentration, while the scores of LV2 mainly separate the three data sets according to their alkoxide concentration. The loadings of LV1, LV2 and LV3 (Figure 8b) can visually be related to the three populations observed in the spectra plot (Figure 8c), explaining almost all the variability contained in the spectra ( $X$-variable). The loadings of LV1 (the latent variable most correlated to the CTX concentration) mainly describe the variability in the spectra caused by changes of CTX concentration (the aromatic band shift and slight decrease shown in Figure 6), although the combination of LV1, LV2, and LV3 is required to give a good estimate of CTX concentration independently of the alkoxide concentration. The color code in Figure $8 \mathrm{c}$ has been linked to the CTX concentration, with blue color representing maximum CTX concentration and red color representing CTX depletion. Dashed vertical lines show the peak maxima for the three analytes (alkoxide at 1637 and $1673 \mathrm{~nm}, \mathrm{CTX}$ at $1666 \mathrm{~nm}$, and Allyl $\mathrm{MgCl}$ at $1647 \mathrm{~nm}$ ). The loadings of LV1 show higher absolute values for the region around the CTX maximum, 

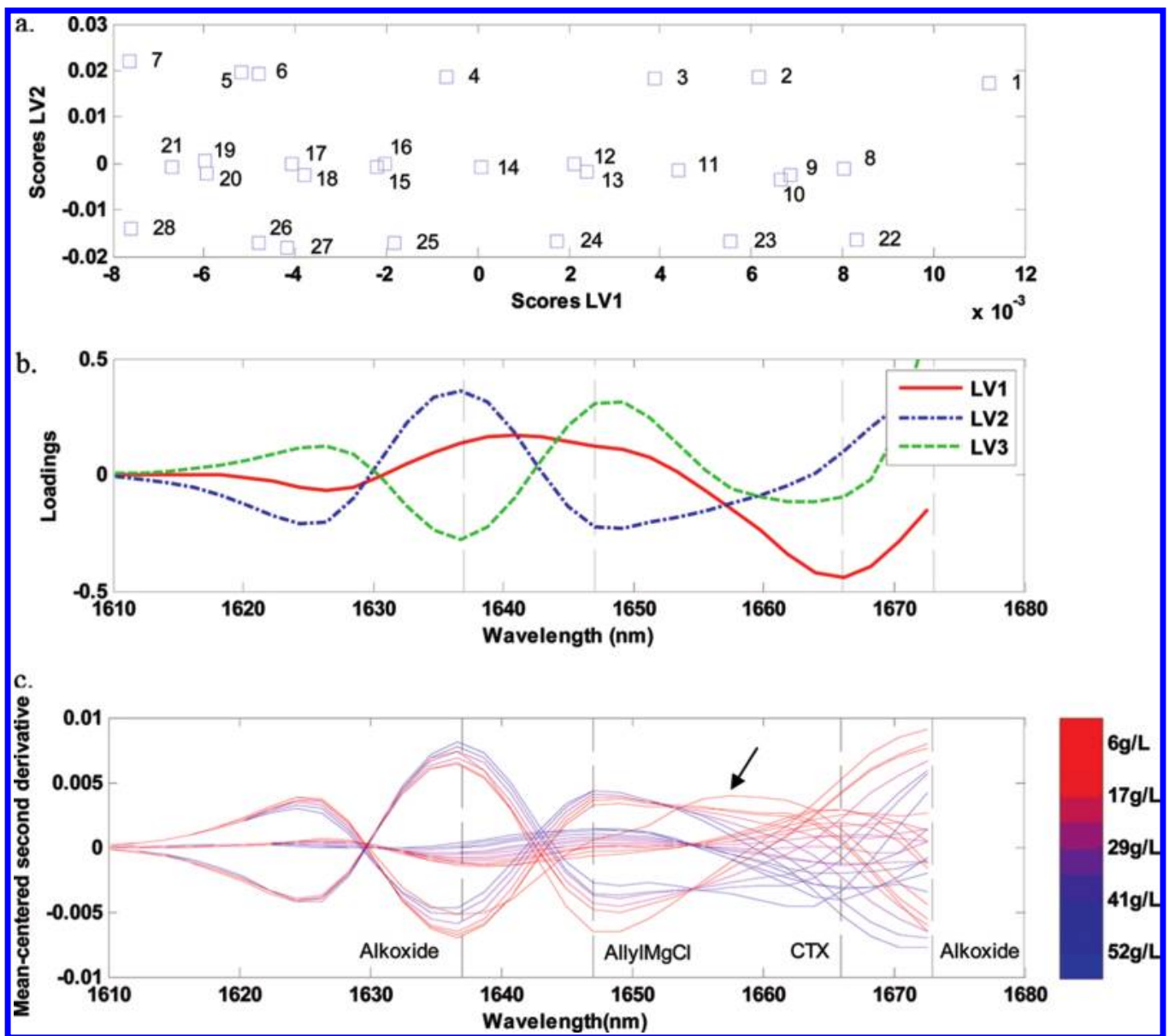

Figure 8. (a) Scores plot for LV1 and LV2, where points 1-7 correspond to calibration data set 1, points 8-21 correspond to calibration data set 2, and points 22-28 correspond to calibration data set 3. (b) Loadings of LV1, LV2, and LV3 in the wavelength interval selected for PLS model construction. (c) Mean-centered second derivative spectra of samples 1-28. The color code is related to CTX concentration as shown in the color bar. An arrow identifies a spectral outlier containing excess Grignard reagent. Dashed vertical lines point to peak maxima of the three important analytes.

where the maximum CTX-related variability is observed. The loadings of LV2 show maxima at the allyl and aromatic alkoxide maxima. Maxima in raw absorbance spectra are seen as minima when second derivative is applied. Since the data is meancentered, at $1637 \mathrm{~nm}$, the spectra lying below zero are lower than the mean and thus contain more alkoxide. Thus, the scores of LV2 are negatively correlated to the alkoxide concentration (points 22-28 have the highest alkoxide concentration and lowest scores). Point 28 is a spectral outlier, which can be observed in Figure 8c (identified with an arrow), since it clearly differs from the rest of the population, with the highest spectral difference occurring at $1647 \mathrm{~nm}$, which is actually the peak maximum for the Grignard reagent. This sample could thereby be identified as having excess Grignard reagent.

3.1.3. PLS Model Validation. The alkylation reaction used for validation was carried out with an initial alkoxide concentration of $180 \mathrm{~g} / \mathrm{L}$. Inline NIR data was gathered every $10 \mathrm{~s}$ and Grignard reagent flow rate was changed in intervals of $5 \mathrm{~min}$ as described in the Materials and Methods section. In the end of each time interval a reference HPLC sample was taken. All the PLS models shown in Table 2 were used to predict CTX concentration for the whole reaction time, and the last NIR sample in each time interval was compared to the reference HPLC measurement. The standard error of prediction (SEP) was calculated according to eq 1. A linear regression (eq 2) was performed relating the NIR model predictions to the reference HPLC measurements, resulting in a bias, slope, and linear regression coefficient $Q^{2}$ reported in Table 2. The model predictions were then corrected for bias and slope deviations (eq 3), obtaining a corrected SEP value,

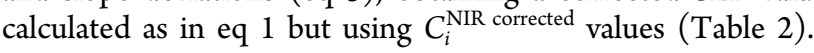

$$
\begin{gathered}
C_{i}^{\mathrm{NIR}}=\text { bias }+ \text { slope } C_{i}^{\mathrm{HPLC}}+\varepsilon_{i} \\
C_{i}^{\mathrm{NIR} \text { corrected }}=\frac{C_{i}^{\mathrm{NIR}}-\text { bias }}{\text { slope }}
\end{gathered}
$$

The 'best' model was considered as the one with lowest SEP, highest $Q^{2}$, lowest absolute bias, slope closest to 1 and lowest SEP corrected. A maximum number of LV of 3 was considered safe in order to maintain robustness and avoid model overfitting. Model 10 was thus considered as a good model, while models 2 and 4 are also expected to be robust thanks to the low number of LV. Interestingly, models 1-14 (those pretreated with $\mathrm{BC}$ or derivatives) all resulted in very low SEP after bias and slope correction (even the highly overfitted model 5), catching perfectly the CTX concentration trend, while the models pretreated with MSC and SNV showed a lower performance. The bias and slope correction is a very simple procedure which may be applied for model maintenance during production. ${ }^{31,37}$ 
Figure 9 shows CTX concentration predictions from model 10 against reference HPLC measurements. The horizontal error

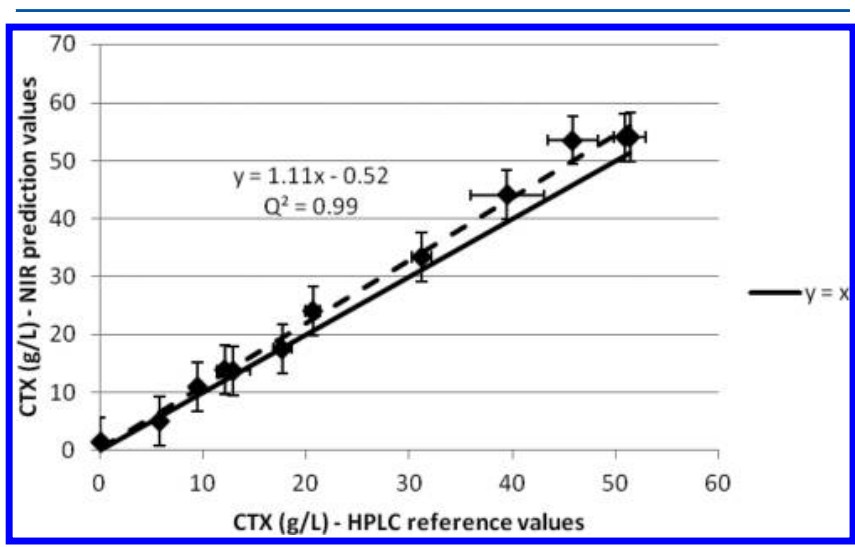

Figure 9. Predicted CTX concentration vs HPLC reference concentration in the validation data set, given by PLS model 10 .

bars represent the standard deviation for the three HPLC measurement replicates, while the vertical error bars are the SEP. Model 10 is especially accurate at low CTX concentrations, which is very positive for the intended use of the PLS model while it shows a slight slope deviation. This deviation is most likely due to a systematic HPLC measurement error occurring during the sample dilution procedure. These dilutions had to be carried out by a person different from the one who performed the dilution work in the calibration experiments, introducing a human source of variability. Furthermore, a different experimental work has shown that the pipet used for dilutions is probably taking a lower volume than it should. This agrees with the fact that the NIR model predictions systematically overestimate with an amount proportional to the CTX HPLC reference concentration (note that all the slope values reported in Table 2 for models $1-14$ are similar and greater than 1 ).

3.2. Process Monitoring Tools. Considering the sytem under study, a process monitoring system should offer as many as possible of the following features:

- Accurate measurement of CTX concentration, with the ability to follow its trend in real time.
- Simple model supervision, maintenance and update, including new spectra or performing simple bias and slope corrections by taking HPLC reference analysis on a periodic basis.

- Provide an approximate concentration value for the alkoxide in real time, in order to calculate product flow rate and determine when a batch of product has been processed.

- Establish an alarm system, indicating system anomalies such as excess of Grignard reagent in the product stream or accumulation of solids in suspension, with potential to cause a reactor blockage.

The alkylation reaction used for validation provided measurements every $10 \mathrm{~s}$ which could be used to simulate a real-time inline monitoring system, while providing information about the dynamics of the reactor. Figure 10 shows the CTX concentration as a response to step changes in the Grignard reagent flow rate into the reactor. Both the original NIR predictions given by model 10 and the corrected values have been plotted, as well as the reference HPLC measurements at the end of each time interval (when steady state was reached). The experiment was planned so that full CTX conversion would be obtained with $2 \mathrm{~mL} / \mathrm{min}$ of Grignard reagent into the reactor, and the flow rate of reagent was regulated so that the model could be validated near the titration point and also with an excess of Grignard reagent. However, the Grignard reagent concentration was lower than that measured the day previous to the experiment, and thus full CTX conversion was only obtained with around $3 \mathrm{~mL} / \mathrm{min}$, indicating that the real Grignard reagent concentration was around $0.67 \mathrm{M}$ or the pump was providing a lower flow rate than expected.

The experiment started with solvent in the reactor (THF). At time 0 , the flow of CTX-saturated alkoxide solution into the reactor was started. After reaching steady state, the flow of Grignard reagent was started at $2 \mathrm{~mL} / \mathrm{min}$ until steady state was reached again. At this point $(t=10 \mathrm{~min})$ the two reactants had been loaded into the reactor, and the flow rate of Grignard reagent was turned off and then increased gradually as shown in Figure 10. Right after increasing the flow rate from 3 to $4 \mathrm{~mL} /$ min, a blockage of the Grignard reagent entrance into the reactor occurred, observed by an increase in the pressure in manometer M2 (Figure 2) above the safe pressure limit of the PTFE tube used for the peristaltic pump. The flow rate of

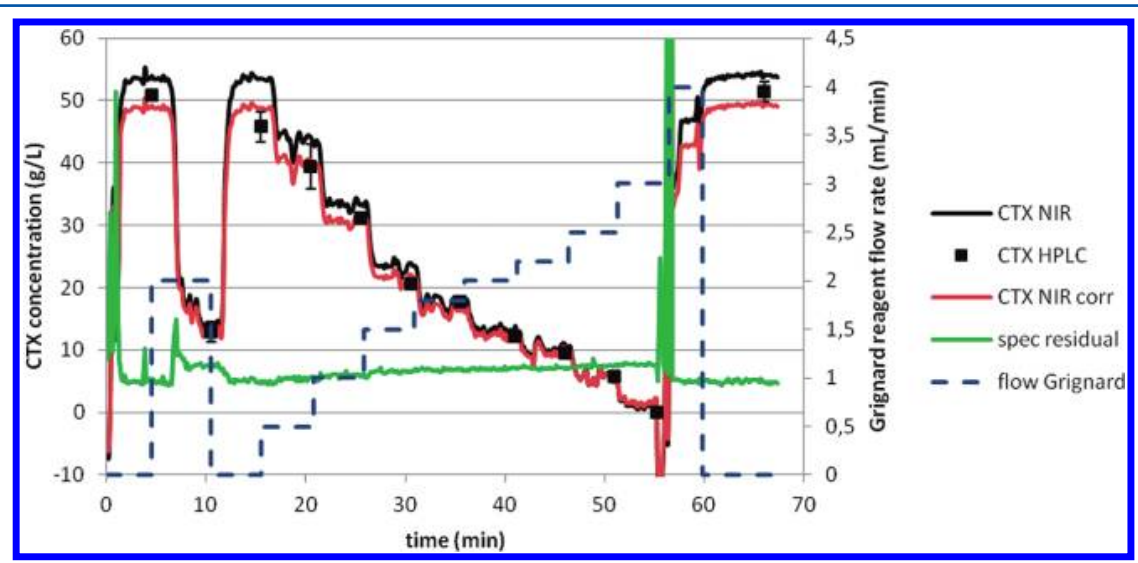

Figure 10. Real-time inline monitoring of the validation alkylation reaction. The original NIR predictions of CTX concentration according to model 10 (CTX NIR), the corrected prediction after bias and slope correction (CTX NIR corr), the CTX HPLC measurements, the spectral residual and the Grignard reagent flow rate are plotted against time. 
Grignard reagent was then turned off (keeping the CTXsaturated alkoxide flow rate on), and the experiment was finished when steady state was achieved.

The CTX concentration profile could be followed in real time, with a high agreement with HPLC reference measurements (even with not corrected predictions) and providing much richer information on the dynamics of the reactor and all the events that occurred during the process than offline HPLC analysis would. The scaled spectral residual for each NIR measurement has also been plotted, calculated as the difference between the measured spectra and the reconstructed spectra according to the PLS model. It provides an excellent way of monitoring when the spectra contain information which was not included in the calibration data set, as well as helping to detect system anomalies. In the first $2 \mathrm{~min}$ of the reaction, the spectral residual is very large, indicating that THF is in the system. The spectral residual is kept at a low level throughout the reaction except after approximately $55 \mathrm{~min}$, when an excess of Grignard reagent was detected at the flow cell. Unfortunately the Grignard reagent excess could not be maintained for a long time due to the aforementioned blockage. The mean-centered second derivative of the spectra obtained throughout all the experiment has been plotted in Figure 11. The spectra obtained

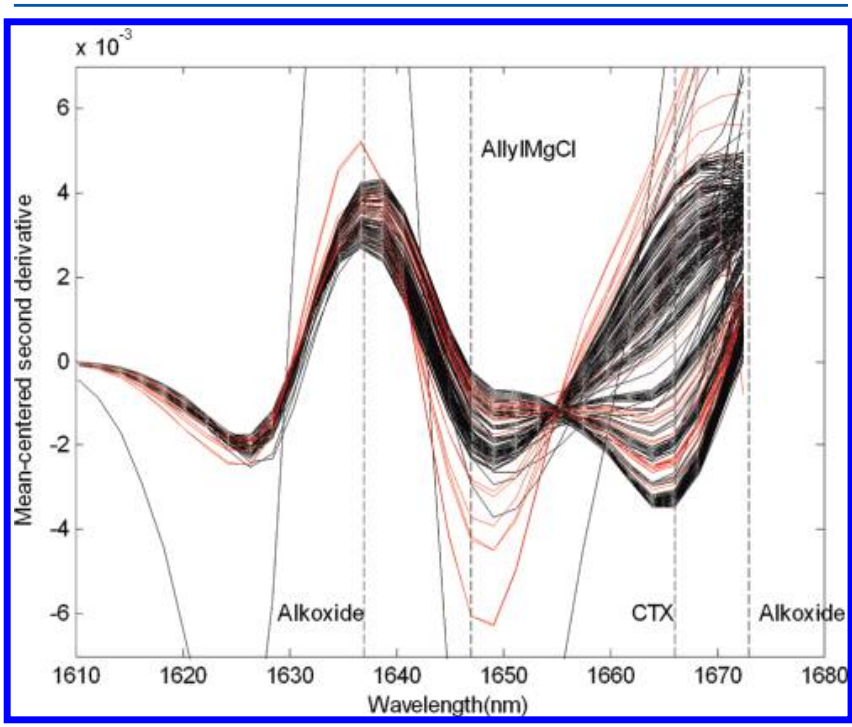

Figure 11. Mean-centered second derivative of the spectra obtained throughout the validation experiment, where spectra from NIR samples between 55 and 60 min have been plotted in red color, assisting in the identification of spectral outliers related to excess Grignard reagent in solution. Dashed vertical lines indicate peak maxima for the important analytes.

between 55 and $60 \mathrm{~min}$ have been plotted in red color. It is possible to distinguish that some of the spectra in this time interval clearly separate from the rest of the population, showing a maximum separation at the AllylMgCl peak maximum, thus indicating that the solution contains excess Grignard reagent. The maximum variability in the spectra occurs at the CTX peak maximum (1666 nm). The spectra having the lowest values at this wavelength correspond to the highest CTX concentration (by comparison with Figure 8c). Therefore, an inspection of the mean-centered second derivative spectra (disregarding PLS model predictions) may be very useful for monitoring the reactor status and supervision of the PLS model performance.
Following the alkylation reaction, the alkoxide must be hydrolyzed in order to produce allylcarbinol, and the organic and aqueous phases generated must be separated. ${ }^{35}$ An excess of Grignard reagent in the alkoxide product stream would react with the acidic water used for hydrolysis, producing propene gas. $^{24}$ This reaction is extremely exothermic, while propene gas in high amounts could cause pressure buildup. Therefore, the hydrolysis reactor should be vented with a pressure relief valve, while pressure increase would be a simple means of detecting excess Grignard reagent in the product stream.

The alkoxide concentration values were determined using a PLS model with the same characteristics as CTX model 10, but with only $2 \mathrm{LV}$. The accuracy of the model is only fair, with a SEP in the order of $20 \mathrm{~g} / \mathrm{L}$ (range $0-300 \mathrm{~g} / \mathrm{L}$ in the calibration data set). This is due to the fact that the calibration data set contained alkoxide values around basically three levels. A calibration could easily be extended to include more variation in the alkoxide concentration but this is out of scope for this work. In an industrial implementation it has also been proposed to use absolute absorption values at wavelengths where no compounds are absorbing, which could be used to determine scattering in the media related to solids in suspension with potential to cause blockages.

3.3. Dynamics of the Alkylation Reactor. The response of the alkylation reactor to the step changes introduced by the Grignard reagent flow rate could be used to obtain a simple model of the reactor dynamics, which is required for the design of the reactor control system. The simplified model proposed neglects the effect of the side entries and reduces the system to an imaginary CSTR reactor in series with a plug flow reactor. The CSTR represents diffusion in the axial direction and a simplistic residence time distribution. The plug flow reactor introduces a time delay into the system. The resulting model is given by eq 4 and eq 5 , where $C_{3}$ is the CTX concentration out of the CSTR reactor, $C_{1}$ is the concentration of CTX in the alkoxide solution into the side-entry reactor, $C_{2}$ is the concentration of Grignard reagent into the reactor, $v_{1}$ is the alkoxide flow rate into the reactor, $v_{2}$ is the Grignard reagent flow rate and $V$ is the volume of the imaginary CSTR. $C_{4}$ is the CTX concentration out of the tubular reactor, with a time delay given by the volume of a tubular reactor $V_{\mathrm{p}}$. The model thus summarizes the dynamics of the reactor into a first-order process with time delay, with a time constant given by eq 6 and a time delay given by eq 7 .

$$
\begin{aligned}
& \frac{\mathrm{d} C_{3}}{\mathrm{~d} t}=\left[\frac{v_{1}}{v_{1}+v_{2}} C_{1}-\frac{v_{2}}{v_{1}+v_{2}} C_{2}-C_{3}\right] \frac{v_{1}+v_{2}}{V} \\
& C_{4}(t)=C_{3}\left(t-\frac{V_{\mathrm{p}}}{v_{1}+v_{2}}\right) \\
& \tau=\frac{V}{v_{1}+v_{2}} \\
& \theta=\frac{V_{\mathrm{p}}}{v_{1}+v_{2}}
\end{aligned}
$$

On the basis of the inline data obtained for the CTX concentration out of the reactor as a response to the step changes performed in $v_{2}$, a nonlinear regression was made to find estimates of the parameters $V, V_{\mathrm{p}}$ and the concentration of Grignard reagent $C_{2}$, which was lower than expected as explained above. Figure 12 shows that the model fits well to the 


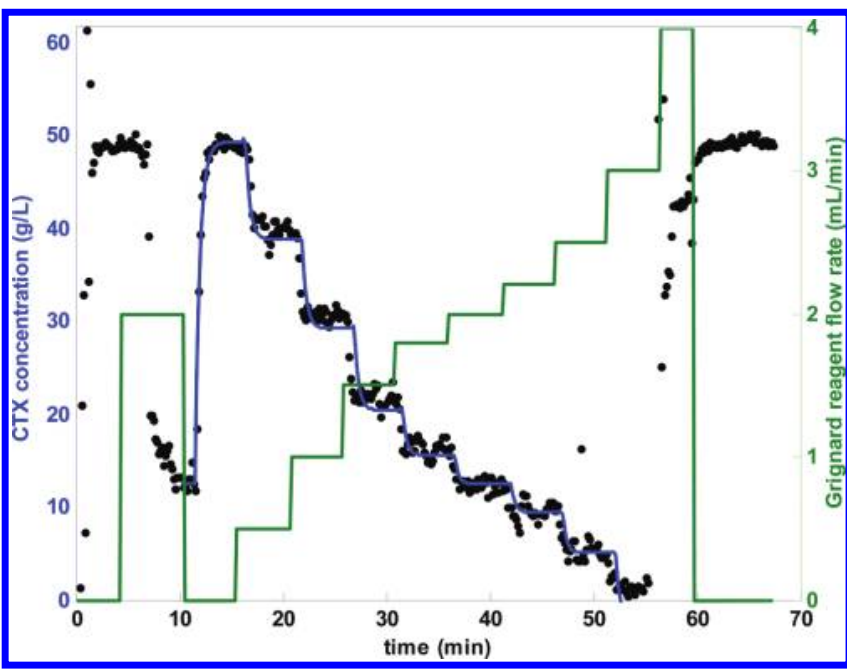

Figure 12. Inline CTX concentrations obtained by NIR spectroscopy (black dots) and model adjustment (blue curve) as a function of the Grignard reagent flow (green curve) into the reactor.

experimental data and results in $V=4.7 \mathrm{~mL}, V_{\mathrm{p}}=11 \mathrm{~mL}$ and $C_{2}=0.7 \mathrm{M}$. For a flow rate $v_{1}=10 \mathrm{~mL} / \mathrm{min}$ and $v_{2}=0-2 \mathrm{~mL} /$ min, the time constant and time delay in the system are $\tau=$ $0.39-0.47 \mathrm{~min}$ and $\theta=0.92-1.1 \mathrm{~min}$, respectively. Therefore, the online data obtained using NIR spectroscopy combined with a dynamic model and the knowledge of a certain input into the system provides information about the dynamics of the system as well as about unknown variables such as the Grignard reagent concentration in this case. In this way, extra measurements such as the Grignard reagent concentration could be unnecessary, or alternatively, the combination of online spectroscopic data with a model of the system could be used to detect system anomalies or monitoring incoherencies, as in this case, a decrease in Grignard reagent concentration.

3.4. Process Control Simulation for the Evaluation of the Monitoring Performance. Chemometricians usually face the problem of optimizing the prediction ability and robustness of the models they develop. However, it is often difficult to determine what the minimum acceptable level of model uncertainty is, as well as how often inline data should be collected. An excessive amount of collected data might lead to data storage problems as well as high computational loads for data processing. A simple approach to address this problem was followed in this work. The dynamic model of the alkylation reactor and the knowledge of the prediction ability of the NIR spectroscopic method $(\mathrm{SEP}=3.2 \mathrm{~g} / \mathrm{L})$ and the sampling time $(5 \mathrm{~s}$, measurements every $10 \mathrm{~s}$ ) were used to simulate the behavior of a feedforward-feedback controller implemented according to the scheme in Figure 3. The flow rate of CTXsaturated alkoxide solution to the reactor, its CTX concentration, and the concentration of Grignard reagent were all set as disturbance variables while the flow rate of Grignard reagent into the reactor was the manipulated variable. Different disturbances were simulated in form of step changes. It was assumed that these disturbances could be measured with a certain random noise (characterized by a bias and a variance) and a sampling time, typical of the instruments which could be used for monitoring of these variables (flow meter, temperature measurement combined with solubility model for prediction of CTX concentration at the inlet, and NIR measurement for prediction of the Grignard reagent concentration). The Simulink model of the process control scheme is shown in Figure 13, while the process control simulation results are plotted in Figure 14. In order to demonstrate the importance of the feedback control loop, the measurement bias for the Grignard reagent concentration was set to $30 \%$. It can be seen that the feedback controller corrects the error introduced by the feedforward controller, making sure that the CTX concentration out of the reactor lies close to the set point (3.5 g/L for CTX concentration). Negative values of concentration simply mean that an excess of Grignard reagent would be found in the outlet of the reactor.

A histogram was made including all the samples measured at the outlet of the reactor for the whole simulation time (20 min). On Figure 15, acceptable output concentration limits were drawn (e.g., in this case, the upper concentration limit was set to $\sim 10 \mathrm{~g} / \mathrm{L}$, and the lowest acceptable limit was set to $0 \mathrm{~g} / \mathrm{L}$ of CTX); the percentage of samples meeting the upper and lower acceptable limits was calculated. For the example shown

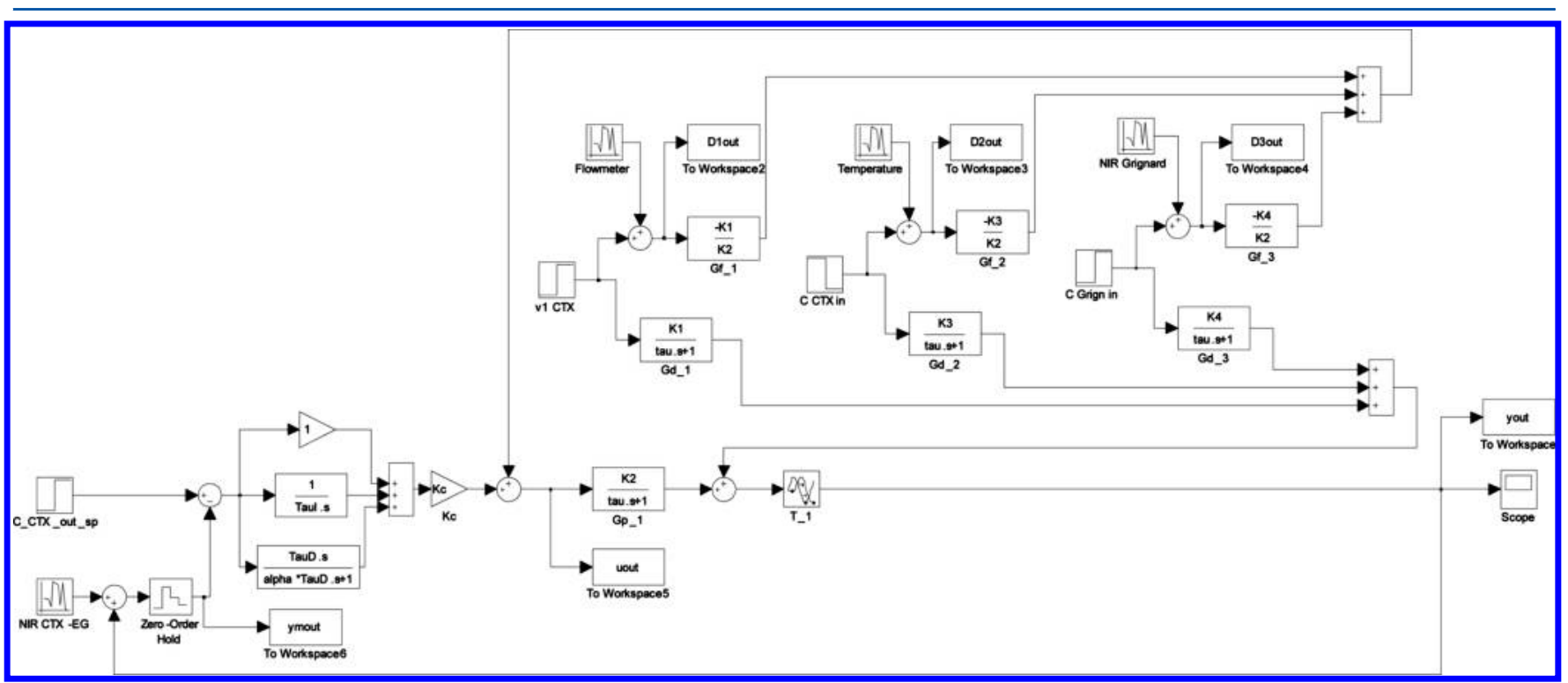

Figure 13. Simulink model used to simulate the feedforward-feedback control loop proposed. 


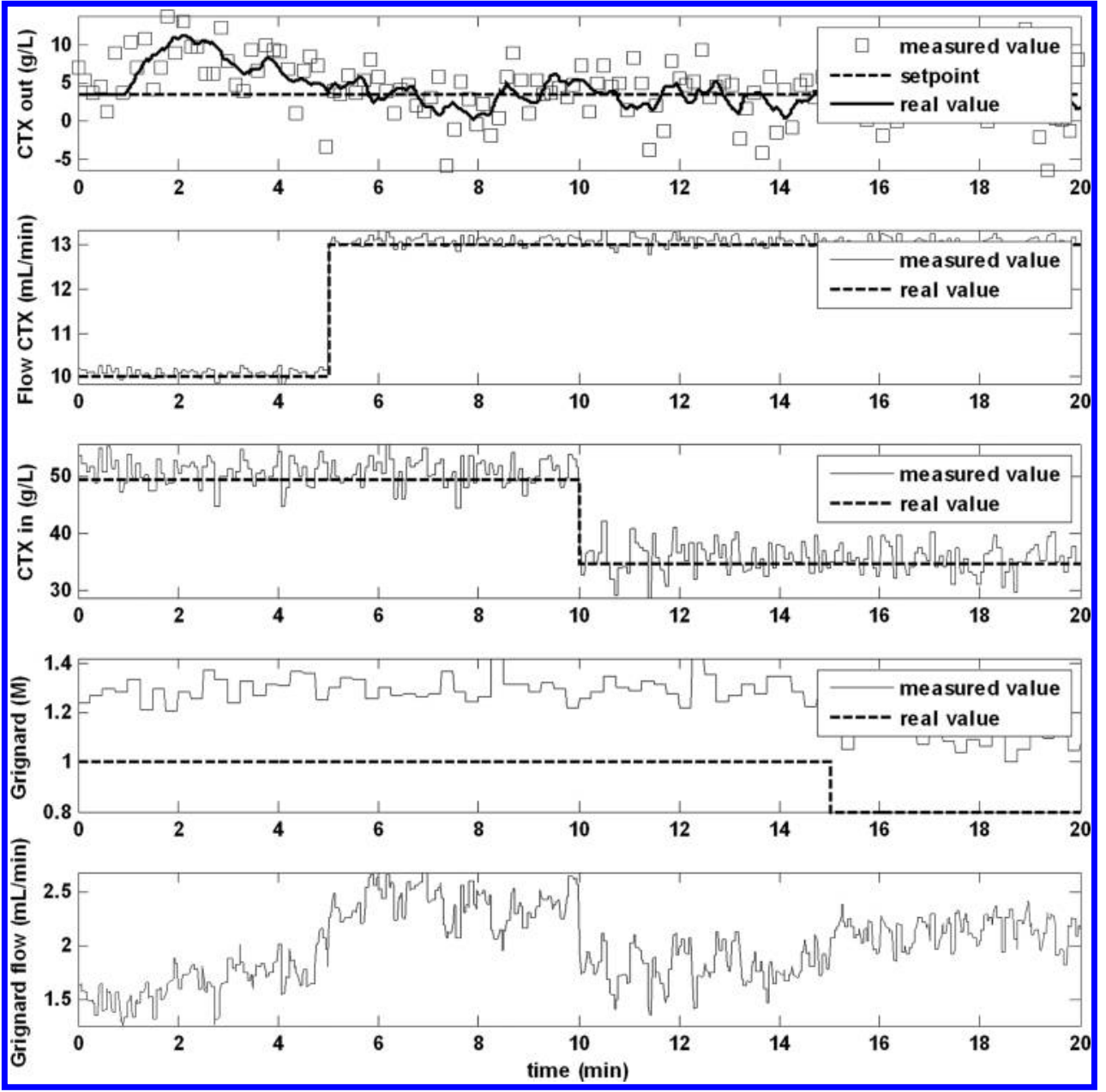

Figure 14. Process control simulation output using $3.5 \mathrm{~g} / \mathrm{L}$ as the set point for CTX concentration. A measurement bias of $30 \%$ in the determination of Grignard reagent concentration was used as an example of a disturbance caused by a measurement anomaly in the feedforward control loop.

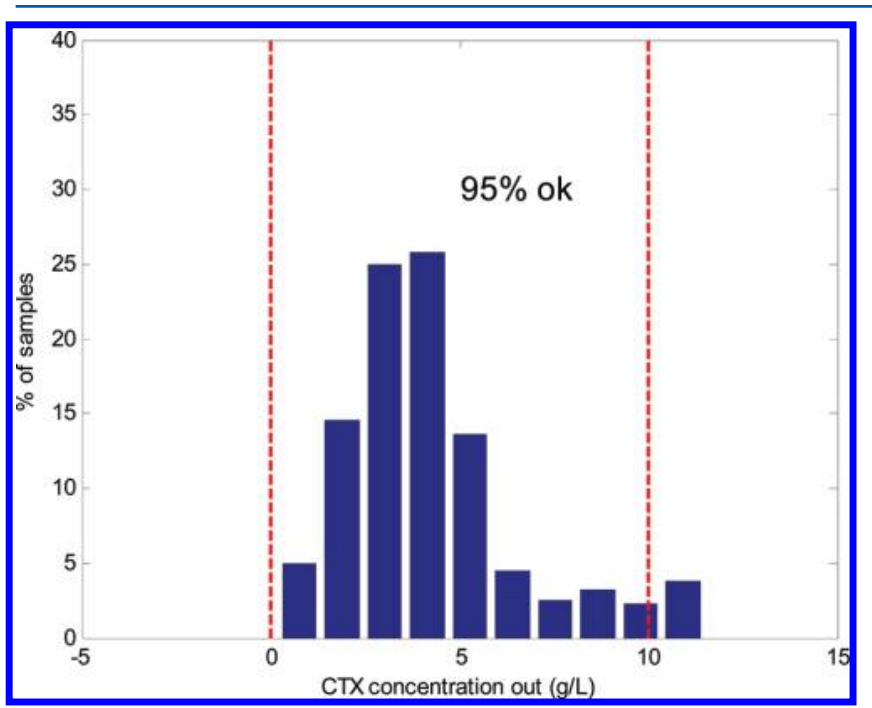

Figure 15. Histogram calculated by including all the NIR samples obtained during the $20 \mathrm{~min}$ of simulated production, where low and high acceptance limits have been drawn (vertical dashed lines).

in Figures 14 and 15, 95\% of the samples lie within the acceptable limits, and only $5 \%$ of them deviate due to the small time it takes for the feedback controller to correct the error introduced by the bias in the Grignard reagent concentration determination. While this simulation was only an example of a possible (quite realistic) scenario, this type of relatively simple tools could be useful for the assessment of different analytical tools, determination of number of measurements and sampling frequencies and evaluation of process control performance. All this information would be very useful for the development of a flexible and controllable design space, where product specifications can be ensured in real time.

\section{CONCLUSIONS}

A system for monitoring and control of a continuous alkylation reaction for the synthesis of an API intermediate using inline NIR spectroscopy data has been developed. The Grignard reaction studied is very sensitive to stoichiometric ratio: an excess of the Grignard reagent results in an impurity formation, while an excess of the ketone substrate involves a yield loss. In order to keep this fast and exothermic reaction under control with low impurity formation, a tubular reactor with up to three side entries controlled by a feedforward-feedback loop was conceived. While the feedforward action results in faster control with little or no time delays, it can only respond to frequent and accurate measurements of disturbance variables. Feedback control is required for correction against nonmeasured variables, measurement bias or detection of system anomalies. An inline NIR spectroscopic method was developed in order to determine ketone substrate concentration remaining in the 
product in real time, while detecting excess of Grignard reagent by analysis of pretreated spectra. These variables are related to system descriptors such as impurity formation (product quality) and yield, and thus contribute to the real time evaluation of the process performance, while paving the way to real time release. The PLS model used to regress the NIR spectroscopic data against HPLC reference measurements was based on three independent experiments at different concentrations of the alkoxide product, obtaining a satisfactory standard error of cross-validation. The model was validated with a fourth alkylation experiment, obtaining good predictions with low bias and slope deviations. The frequent measurements obtained during the last alkylation experiment, where the Grignard reagent flow rate was manipulated to obtain different responses, provided the opportunity to develop a simple model to describe the reactor dynamics. This model was then used to tune a simple PI controller and simulate the process control performance. Finally, the simulation output could be used to evaluate the quality of the different analyzers in the system and decide the frequency for data collection. Operation at Lundbeck A/S has demonstrated that the continuous alkylation reaction can be performed with low impurity formation and high yields.

\section{AUTHOR INFORMATION}

\section{Corresponding Author}

*Telephone: +45 45252970. Fax: +45 45932906. E-mail: kvg@ kt.dtu.dk.

\section{ACKNOWLEDGMENTS}

We thank the Technical University of Denmark and Lundbeck A/S for technical and financial support, Q-Interline A/S for support with the NIR spectrophotometer, and the KT workshop and Mette Larsen for technical assistance during the experimental work. The students who participated in related activities are also gratefully acknowledged.

\section{ADDITIONAL NOTE}

${ }^{a}$ Avoiding Grignard reagent excess minimizes byproduct formation as explained above. Although it would be conceivable to setup a control strategy based on a direct measurement of the main byproduct (using inline NIR spectroscopy data), it is in practice simpler to monitor the main components in solution (alkoxide, CTX and Grignard reagent) and avoid the conditions at which it is known that the byproduct is formed. Due to the molecular similarities of the different analytes, the NIR absorption spectra are dominated by the components present in the highest concentration.

\section{REFERENCES}

(1) Behr, A.; Brehme, V. A.; Ewers, C. L. J.; Grön, H.; Kimmel, T.; Küppers, S.; Symietz, I. Eng. Life Sci. 2004, 4, 15.

(2) Jiménez-González, C.; Poechlauer, P.; Broxterman, Q. B.; Yang, B.; am Ende, D.; Baird, J.; Bertsch, C.; Hannah, R. E.; Dell'Orco, P.; Noorman, H.; Yee, S.; Reintjens, R.; Wells, A.; Massonneau, V.; Manley, J. Org. Process Res. Dev. 2011, 15, 900.

(3) Kang, L.; Chung, B. G.; Langer, R.; Khademhosseini, A. Drug Discoverv Todav 2008, 13, 1 .

(4) Schaber, S. D.; Gerogiorgis, D. I.; Ramachandran, R.; Evans, J. M. B.; Barton, P. I.; Trout, B. L. Ind. Eng. Chem. Res. 2011, 50, 10083.

(5) Webb, D.; Jamison, T. F. Chem. Sci. 2010, 1, 675.

(6) LaPorte, T. L.; Wang, C. Curr. Opin. Drug Discovery Dev. 2007, 10, 738 .

(7) Plumb, K. Chem. Eng. Res. Des. 2005, 83, 730.
(8) Pollet, P.; Cope, E. D.; Kassner, M. K.; Charney, R.; Terett, S. H.; Richman, K. W.; Dubay, W.; Stringer, J.; Eckert, C. A.; Liotta, C. L. Ind. Eng. Chem. Res. 2009, 48, 7032.

(9) U.S. Food and Drug Administration (FDA), PAT Guidance, 2004.

(10) Hartman, R. L.; Jensen, K. F. Lab Chip 2009, 9, 2495.

(11) Kockmann, N.; Roberge, D. M. Chem. Eng. Technol. 2009, 32, 1682.

(12) Vervaet, C.; Remon, J. P. Chem. Eng. Sci. 2005, 60, 3949.

(13) Barthe, P.; Guermeur, C.; Lobet, O.; Moreno, M.; Woehl, P.; Roberge, D. M.; Bieler, N.; Zimmermann, B. Chem. Eng. Technol. 2008, 31, 1146.

(14) Jensen, K. F. Chem. Eng. Sci. 2001, 56, 293.

(15) Roberge, D. M.; Bieler, N.; Mathier, M.; Eyholzer, M.; Zimmermann, B.; Barthe, P.; Guermeur, C.; Lobet, O.; Moreno, M.; Woehl, P. Chem. Eng. Technol. 2008, 31, 1155.

(16) Hartman, R. L.; Sahoo, H. R.; Yen, B. C.; Jensen, K. F. Lab Chip 2009, 9, 1843.

(17) Kralj, J. G.; Sahoo, H. R.; Jensen, K. F. Lab Chip 2007, 7, 256.

(18) Hartman, R. L.; Naber, J. R.; Buchwald, S. L.; Jensen, K. F. Angew. Chem., Int. Ed. 2010, 49, 899.

(19) Sahoo, H. R.; Kralj, J. G.; Jensen, K. F. Angew. Chem. 2007, 119, 5806.

(20) Mendorf, M.; Nachtrodt, H.; Mescher, A.; Ghaini, A.; Agar, D. W. Ind. Eng. Chem. Res. 2010, 49, 10908.

(21) Kockmann, N.; Gottsponer, M.; Roberge, D. M. Chem. Eng. J. 2011, 167, 718 .

(22) Hartman, R. L.; Naber, J. R.; Zaborenko, N.; Buchwald, S. L.; Jensen, K. F. Org. Process Res. Dev. 2010, 14, 1347.

(23) Roberge, D. M.; Ducry, L.; Bieler, N.; Cretton, P.; Zimmermann, B. Chem. Eng. Technol. 2005, 28, 318.

(24) Silverman, G. S.; Rakita, P. E. Handbook of Grignard Reagents; CRC Press: New York, 1996.

(25) Müller Christensen, K.; Jønch Pedersen, M.; Dam-Johansen, K.; Lønberg Holm, T.; Skovby, T.; Kiil, S. Chem. Eng. Sci. 2011, DOI: $10.1016 /$ j.ces.2011.12.002.

(26) McMullen, J. P.; Jensen, K. F. Annu. Rev. Anal. Chem. 2010, 3, 19.

(27) McMullen, J. P.; Jensen, K. F. Org. Process Res. Dev. 2010, 14, 1169.

(28) McMullen, J. P.; Jensen, K. F. Org. Process Res. Dev. 2011, 15, 398.

(29) Loebbecke, S.; Ferstl, W.; Panić, S.; Türcke, T. Chem. Eng. Technol. 2005, 28, 484.

(30) Ferstl, W.; Klahn, T.; Schweikert, W.; Billeb, G.; Schwarzer, M.; Loebbecke, S. Chem. Eng. Technol. 2007, 30, 370.

(31) Roggo, Y.; Chalus, P.; Maurer, L.; Lema-Martinez, C.; Edmond, A.; Jent, N. I. Pharm. Biomed. Anal. 2007, 44, 683.

(32) Carter, C. F.; Lange, H.; Ley, S. V.; Baxendale, I. R.; Wittkamp, B.; Goode, J. G.; Gaunt, N. L. Org. Process Res. Dev. 2010, 14, 393.

(33) Wiss, J.; Länzlinger, M.; Wermuth, M. Org. Process Res. Dev. 2005, 9, 365 .

(34) Wiss, J.; Ermini, G. Org. Process Res. Dev. 2006, 10, 1282.

(35) Cervera-Padrell, A. E.; Morthensen, S. T.; Lewandowski, D. J.; Skovby, T.; Kiil, S.; Gernaey, K. V. Continuous hydrolysis and L-L phase separation of an active pharmaceutical ingredient intermediate using a mini-scale hydrophobic membrane separator. Org. Process Res. Dev. Submitted 2012.

(36) Wold, S.; Sjöström, M.; Eriksson, L. Chemom. Intell. Lab. Syst. 2001, 58, 109.

(37) Bakeev, K. A. Process Analytical Technology; Blackwell Publishing: India, 2005.

(38) Petersen, N.; Ödman, P.; Padrell, A. E. C.; Stocks, S.; Lantz, A. E.; Gernaey, K. V. Biotechnol. Prog. 2010, 26, 263.

(39) Osborne, B. G.; Fearn, T.; Hindle, P. H. Practical NIR Spectroscopy with Applications in Food and Beverage Analysis; Longman Scientific \& Technical: Harlow, 1993. 